\title{
Does morphological complexity affect word segmentation? Evidence from computational modeling
}

Georgia Loukatou ${ }^{1}$, Sabine Stoll $^{2}$, Damian Blasi ${ }^{2,3}$, and Alejandrina Cristia ${ }^{1}$

${ }^{1}$ LSCP, Département d'études cognitives, ENS, EHESS, CNRS, PSL University, 75005 Paris, France

${ }^{2}$ Department of Comparative Language Science, Language Development Laboratory, University of Zurich, 8032 Zurich, Switzerland

${ }^{3}$ Center for the Interdisciplinary Study of Language Evolution, ISLE, University of Zurich, 8032 Zurich, Switzerland

Correspondence: georgialoukatou@gmail.com

Declarations of interest: none

GL and AC acknowledge financial and institutional support from the Agence Nationale de la Recherche (ANR-14-CE30-0003 MechELex, ANR-10-IDEX-0001-02 PSL*, and ANR-10-LABX-0087 IEC) and the J.S. McDonnell Foundation.

SS and DB acknowledge financial support from the European Union's Seventh Framework Programme (FP7/2007-2013) under grant agreement 615988 "Acquisition processes in maximally diverse languages: min(d)ing the ambient language"(PI Sabine Stoll). 


\title{
Does morphological complexity affect word segmentation? Evidence from computational modeling
}

\begin{abstract}
How can infants detect where words or morphemes start and end in the continuous stream of speech? Previous computational studies have investigated this question mainly for English, where morpheme and word boundaries are often isomorphic. Yet in many languages, words are often multimorphemic, such that word and morpheme boundaries do not align. Our study employed corpora of two languages that differ in the complexity of inflectional morphology, Chintang (Sino-Tibetan) and Japanese (in Experiment 1), as well as corpora of artificial languages ranging in morphological complexity, as measured by the ratio and distribution of morphemes per word (in Experiments 2 and 3). We used two baselines and three conceptually diverse word segmentation algorithms, two of which rely purely on sublexical information using distributional cues, and one that builds a lexicon. The algorithms' performance was evaluated on both word- and morpheme-level representations of the corpora.

Segmentation results were better for the morphologically simpler languages than for the morphologically more complex languages, in line with the hypothesis that languages with greater inflectional complexity could be more difficult to segment into words. We further show that the effect of morphological complexity is relatively small, compared to that of algorithm and evaluation level. We therefore recommend that infant researchers look for signatures of the different segmentation algorithms and strategies, before looking for differences in infant segmentation landmarks across languages varying in complexity.
\end{abstract}

Keywords: cross-linguistic variation, computational modeling, statistical learning, word segmentation, language acquisition, morphology, artificial language

\section{Introduction}

Typically-developing children acquire language effortlessly and implicitly in the first years of their life. They process linguistic material provided by their caregivers and 
others around them using robust learning mechanisms that do not require meta-linguistic awareness. Infants begin learning the building blocks of language, i.e., words or morphemes, from very early on, achieving a comprehension vocabulary of hundreds of words by two years of age (Bates et al., 1994). More precisely, during the first year of life, infants might build up a proto-lexicon storing candidate phonological forms (often called wordforms), forms that they have found in their spoken input, without necessarily learning what the forms' meaning or grammatical functions are (Ngon et al., 2013). Here, we report on a series of computational experiments that seek to shed light on the specific processes that young language learners could use when segmenting the incoming speech signal into word-like forms. Setting aside how infants learn the meaning and the grammatical function of these forms, we focus on how infants could find the phonological forms of those minimal meaningful recombinable units (be it words or morphemes), and how they can break up the sentences they hear - that is, how they insert "mental pauses" that separate a sentence into its component units.

\subsection{From cues to algorithms}

To break up the speech stream into its component units, infants can use a variety of algorithms, each of which requires specific cues. Different cues may contribute to segmentation.

We can liken an algorithm to a recipe to be followed: it states the steps that the learner or listener takes. In this metaphor, cues are the ingredients in the recipe - they are pieces of information that the learner looks for in the input if and only if those pieces of information are recruited by the algorithm. For instance, infants could start by breaking up the stream into sentences by using pauses, intonation, and preboundary lengthening (Shukla, White \& Aslin, 2011) - although this would not take them very far as they still need to insert mental pauses between all the words (or morphemes) inside the sentence. To the latter end, they could use two language-general cues that are present in the phonetic signal: coarticulation (Norris, McQueen, Cutler \& Butterfield, 1997), and constraints on stranded material (E. K. Johnson \& Jusczyk, 
2001). The recipe based on coarticulation would require infants to have learned all the phonemes in their language, and to have learned that a given phoneme can be shifted slightly by neighboring phonemes, and further that this shift is greater within words than across word boundaries. Although this algorithm is complex, there exists a laboratory study showing that, in ideal conditions, infants do employ coarticulation when finding the edges of words (Norris et al., 1997). As for constraints on stranded material, another laboratory experiment showed that infants did not insert mental pauses in the sentences they heard if doing so would have left an ill-formed syllable (E. K. Johnson \& Jusczyk, 2001).

In addition, other algorithms can be identified when infants learn other statistical properties present in their input, and then use this information to cut up sentences into smaller parts. Previous laboratory work has shown that, even in the first year, infants use words (Bortfeld, Morgan, Golinkoff \& Rathbun, 2005 Mersad \& Nazzi, 2012), syllable sequences (Black \& Bergmann, 2017), and phone sequence patterns (Mattys \& Jusczyk, 2001) as segmentation cues. Cutting up a sentence when the infant recognizes a word, or a low-probability sequence, are strategies that should work on all languages, since all languages have words and morphemes (which are repeating units that could be memorized by the child) and sequence patterns in the form of phonotactics. In other

\footnotetext{
${ }^{1}$ Infants could also learn other regularities that help mostly in some languages. For example, English learners use stress to segment, cutting just before a stressed syllable, at around 7.5 months (Jusczyk, Houston \& Newsome, 1999). However, they abandon this strategy by 10.5 months, which is fortunate because such a strategy causes mis-segmentation of all words that do not start with a strong syllable (like guitar; Jusczyk et al., 1999). Infants learning Turkish, but not German learners, use vowel harmony cues when segmenting words (Van Kampen et al., 2008). We do not discuss these strategies in this paper because they are language-specific: stress mostly helps in languages with fixed stress, vowel harmony in languages with vowel harmony. Since our paper is among the first to study potential differences across languages, it seems important to start with strategies that can be used in all human languages (such as the ones mentioned above, cutting up a sentence when recognizing a word, or when observing a low-probability sequence). Also, note that even in such language-general strategies, infants still need to know about how it is implemented in their native language; leading some authors to classify the same strategy as language-specific or language-general (e.g. stress, Endress and Hauser,
} 
words, the infant would add a mental boundary before and after a recognized word, separating the phrase in two word-like chunks. Similarly, the infant would add a mental boundary in the middle of a low-probability sequence, separating the two chunks.

Thus, a variety of algorithms (or recipes for inserting mental pauses within sentences) have been documented in controlled laboratory experiments. Although this work is crucial because it proves that certain algorithms are active in the young child, it does not tell us several things. First, it does not tell us whether these strategies, which are carefully isolated in the lab, are actually functional in the real world (Keren-Portnoy, Vihman \& Fisher, 2019). Second, it does not tell us, if they were functional, whether they would be successful in retrieving at least some word or morpheme units from infants' everyday input in various languages. These strategies were also largely motivated by English and other European languages, which offer a narrow perspective of the nature and the complexity of words and morphemes that children learning other languages might encounter. Our work seeks to address the second limitation; we return to the first limitation in the Discussion.

How can we know whether an algorithm could succeed when provided with realistic input? One approach is to use computational modeling. Computational models are the only way to realistically study learnability in vitro, controlling for factors that are impossible to control in an experiment with human infants. Like infants, these computational models learn in an unsupervised manner, meaning that they do not have access to any kind of feedback (i.e., external information on whether they are doing well or poorly) nor to other information the infant would not have access to (e.g., a syntactic parse or detailed meaning of the sentence).

Computational modeling aiming to break up sentences into its component units has explored three classes of algorithms: lexical, sublexical, and baseline. These classification terms have been used in literature predating our paper (e.g., Bernard et al., 2018) and popularized in previous reviews (e.g. Brent, 1997). Therefore, we adopted them for this study. However, different terms can also be used to convey their 2010: Skoruppa et al., 2009 phonotactics, Blanchard, Heinz and Golinkoff, 2010. 
functions e.g. lexical algorithms could also be classified as global versus the local sublexical ones.

Algorithms in the lexical class aim to build a proto-lexicon of forms. They may try to find the most economical system of minimal units needed to reproduce the input corpus (e.g., Brent and Cartwright, 1996), or alternatively they memorize sound sequences, for instance those that are encountered in isolation (e.g., Monaghan and Christiansen, 2010). We know that young infants have an algorithm like this one in their toolkit thanks to Bortfeld et al. (2005). In that study, infants heard phrases like 'The girl laughed at Mommy's feet.' At test, they demonstrated recognition of the word 'feet', showing that they had used the familiar word 'Mommy' to insert a "mental break" which left 'feet' on its own. (Infants didn't recognize the word 'feet' after familiarization to sentences like 'The girl laughed at Tommy's feet.') We will discuss below that previous modeling work has typically evaluated segmentation considering word breaks, and not morpheme breaks. This does not mean that lexical algorithms necessarily segment at the word level; in fact, particularly algorithms that try to find the most economical system of minimal units may actually find that the optimal solution is to store morphemes, and not full words.

Algorithms in the sublexical class aim instead to find local cues allowing the learner to insert mental pauses (e.g., Saksida, Langus and Nespor, 2017). These local cues could be unusual sound or syllable sequences. Experimental research has shown that this strategy is active in young infants by composing artificial languages in which only syllable sequences (Saffran, Aslin \& Newport, 1996) or sound sequences (Mattys, Jusczyk, Luce \& Morgan, 1999) signal where the mental pauses might be. As with the lexical algorithms, we would like to stress that even though previous computational models building on local approaches have evaluated segmentation at the word level (e.g. Daland and Pierrehumbert, 2011; Gervain and Erra, 2012; Saksida et al., 2017), it is entirely plausible that distributional statistics will result in morpheme breaks being discoverable.

Finally, previous literature has sometimes used word segmentation baselines to 
evaluate the performance of algorithms (Çöltekin, 2011; Lignos, 2012; Venkataraman, 2001). Baselines represent the simplest strategies possible; for example, treating each basic minimal unit (phoneme or syllable) as a word, or treating whole utterances as words. Although we will treat these strategies as baselines in this paper, some have actually proposed the latter as a real strategy infants employ. In particular, Keren-Portnoy et al. (2019) propose that, in realistic conditions, 12-month-olds will only retain words presented in isolation, and not those presented in sentence frames, suggesting that only the former can generate a long-term proto-lexicon (see also Brent and Siskind, 2001 for converging evidence). To our knowledge, the strategy of segmenting each syllable separately has not been proposed for infants (a point to which we return in the Discussion).

\subsection{Cross-linguistic performance}

It has been proposed that language acquisition may not be a homogeneous process, identical in children regardless of the language they are acquiring, but instead that the acquisition process may vary across typologically diverse languages as a function of their grammatical structures (Slobin, 1985). However, the proportion of languages whose acquisition is represented in the literature is low (e.g. Stoll, 2015: Stoll and Lieven, 2014), and the majority of papers on first language development is on English (Slobin, 2014). This sampling bias is problematic because English is not an "average" language, particularly in terms of the properties that may influence segmentation. Most English words have few or no morphemes other than the stem (Aikhenvald, 2007): the maximum number of (inflectional) morphemes per word in English is 3 (Stoll, Mazara \& Bickel, 2017), which is on the lower end of the typological range (Bickel \& Nichols, 2007, 2013b). In English, word, morpheme, and syllable boundaries usually coincide (DeKeyser, 2005; see also Discussion, regarding performance of the baseline algorithm that segments at every syllable edge).

Many other languages are characterized by rich inflectional morphology so that they often feature multi-morphemic words. For example, Turkish has a rich 
concatenative inflectional morphology (Bickel \& Nichols, 2013a, 2013b; Ketrez \& Aksu-Koç, 2009). Others are extremely complex such as the polysynthetic language Chintang (a Sino-Tibetan language spoken in the Himalayas of Eastern Nepal, Stoll et al., 2017) and Eskimo-Aleut languages such as Inuktitut (Allen, 1996 Bickel \& Nichols, 2013b). There is no common agreement on how to measure morphological complexity cross-linguistically (cf. Bane, 2008, Bentz, Soldatova, Koplenig and Samardžić, 2016; McWhorter, 2001; Miestamo et al., 2008; Shosted, 2006). Nonetheless, the goal of this paper is not to look at all aspects of morphological complexity, but to focus on one that seems particularly relevant in the study of segmentation, namely inflectional complexity, which means that content words will have more or fewer affixes, and thus that the boundaries of words and morphemes will align more or less well. In this paper, we speak exclusively of morphological complexity in terms of inflectional synthesis as defined by Bickel and Nichols (2013b): number of inflectional categories (agreement, tense/aspect/mood, evidentials/miratives, status, polarity, illocution, voice) expressed (the definition is drawn from Bickel \& Nichols, 2013b for WALS classification).

Computational modeling work has started to investigate word segmentation in various languages (Batchelder, 2002, Blanchard et al., 2010, Caines, Altmann-Richer \& Buttery, 2019 Daland, 2009; Fleck, 2008; Fourtassi, Börschinger, Johnson \& Dupoux, 2013; Kastner \& Adriaans, 2017; Pearl \& Phillips, 2018; Saksida et al., 2017). Providing a thorough overview of their findings is beyond the scope of the present study, but we would like to highlight that most previous work attempts to check how a given algorithm performs cross-linguistically to argue for the validity of the algorithm the authors of those studies proposed, rather than to understand whether language properties affect segmentation in a systematic way (e.g., Batchelder, 2002; Boruta, Peperkamp, Crabbé and Dupoux, 2011; M. Johnson, 2008, Pearl and Phillips, 2018. Phillips and Pearl, 2014a). Exceptions include studies that try to explain away cross-linguistic differences on the basis of corpus characteristics (e.g., Caines et al., 2019; Fourtassi et al., 2013), and work assessing the effect of prosodic and syntactic 
structure such as head direction (saliently, Gervain and Erra, 2012, Saksida et al., 2017), or the effects of input representation (Kastner \& Adriaans, 2017). However, these factors are orthogonal to the present study (i.e., they are not necessarily confounded with morphological complexity). Therefore, they will not be discussed any further.

\subsection{Goal of segmentation}

The current standard for modeling studies is to evaluate segmentation algorithms on the word level (Daland, 2009). However, we submit that evaluation at the word level alone is insufficient to evaluate both the computational feasibility and the empirical soundness of the algorithms when confronted with languages with non-trivial morphology.

To begin with, there are at least three notions of "word": orthographic word, grammatical word, and prosodic word. According to Haspelmath (2011), orthographic spaces are to some extent guided by language structure, even though spelling can be purely conventional in some cases. Grammatical words are units defined by morphosyntactic criteria, such as cohesiveness, fixed internal order, and conventional meaning. Phonological words are units usually defined by phonological criteria, such as segmental and prosodic features like stress (Dixon \& Aikhenvald, 2002).

Words are not the only meaningful, recombinable units that may be found in running speech. Morphemes can be defined as the minimal meaningful units. Moreover, morphemes and words are not homogeneous classes. For example, functional elements make up a class that cuts across words and morphemes, containing both function words (words expressing grammatical or structural relationship with other words in the sentence) and affixes. Although these definitions seem easy in the abstract, there is no single and standard definition across languages of any of these levels (Bickel \& Nichols, 2007; Bickel \& Zúñiga, 2017).

Is there evidence of what units infants are finding when they segment sentences? Carefully reading experimental evidence, we found that some of it suggests that infants can segment phonological words (E. K. Johnson \& Jusczyk, 2001) as well as morphemes 
(Marquis \& Shi, 2015, Mintz, 2013) out of running speech. Furthermore, they can segment functional elements early on (Hallé, Durand \& de Boysson-Bardies, 2008; Höhle \& Weissenborn, 2003: Marquis \& Shi, 2015; Mintz, 2013; Shi \& Gauthier, 2005, Shi \& Lepage, 2008; Shi, Marquis \& Gauthier, 2006), but this work has not discussed clearly whether these functional elements are best described as words or morphemes. The majority of this research looks at what may be described as functional words (typically determiners, with the exception of Höhle and Weissenborn, 2003, who looked at prepositions). Two papers looked at verbal affixes, namely Mintz (2013), who demonstrated that English-learning 15 month-olds will strip -ing from verbs but not non-verbs; and Marquis and Shi (2015), who reported that 14-month-old French learners familiarized with a novel verb with one affix will nonetheless recognize this verb at test when it is followed by a different affix. Although evidence is scant, we believe it is sufficient to entertain the possibility that infants break segments up not at the word level, but rather at the morpheme level.

On the modeling side, most previous work has evaluated segmentation on orthographic words. In fact, since child-centered corpora are rarely annotated at the level of morphemes, previous computational work looking at infant word segmentation has never quantitatively evaluated performance on the morpheme level (cf. M. Johnson, 2008, but see work directly aiming at unsupervised or weakly supervised morphological segmentation e.g. Ruokolainen et al., 2016). When morphemes are discussed, this is typically in the context of a qualitative evaluation.

In a nutshell, to model how learners break up sentences into smaller units, evaluating computational modeling results at the level of both words and morphemes is a more informative approach.

\subsection{Key Questions and Predictions}

The key questions motivating this study are:

1. Do languages which vary in morphological (inflectional) complexity differ in segmentability? 
2. How large is this effect, compared to others - notably differences across algorithms and as a function of whether evaluation is done at the level of words or morphemes?

In order to answer these questions, we attempted to accurately measure effect sizes (Coe, 2002). This is the first study putting factors next to each other in order to assess their importance. We wanted to establish how large the language effect was, in order to be able to provide informed predictions to be tested through infant experiments. We thus compared the segmentability difference as a function of language complexity against two other factors, which we call level and algorithm. Level refers to whether segmentation is evaluated at the level of words or morphemes. Algorithm refers to the specific strategy employed during segmentation (the algorithms are described in Section 2.1.4). We put our factors (language, algorithm, level) next to each other to assess their relative importance.

Looking specifically into algorithms and levels is an informative way to study segmentability differences of languages differing in morphology. Given that the process of segmentation involves cutting up sentences into units, languages in which morphemes are isomorphic with words may be easier to segment than languages in which words tend to have multiple morphemes ${ }^{2}$ To begin with, surface statistics (recruited by sublexical algorithms) could be less informative across morpheme boundaries within words than across word boundaries (e.g., considering the phrase "the doggy", perhaps a dip in probabilities between sequences like "the" and "dog" is more likely than that between "dog" and "gy").

Additionally, the lexicon of languages with higher inflectional complexity may also differ from that of simpler languages in ways that may affect the cues employed by lexical algorithms. Specifically, languages varying in morphological complexity differ in

\footnotetext{
${ }^{2}$ There could also be phonological factors confounded with morphological complexity, such as word length and segmentation ambiguity. In analyses presented in the online supplementary material (supplementary_material.pdf, section "Proximal causes", pp. 6-9), we show that this is not the case, because these factors do not explain away the language effects we do document.
} 
the frequency of lexical units (words and morphemes). Corpora of morphologically rich languages such as Chintang contain fewer repetitions of each word type, as well as a higher proportion of hapaxes - forms that occur only once - than languages with little morphology (Stoll et al., 2017). For example, there was a higher proportion of hapaxes in a Japanese than an English corpus, and a lower likelihood of correct identification by a lexicon-building algorithm for hapaxes than words with more repetitions (Boruta et al., 2011).

Both lexical and sublexical algorithms might detect word parts such as morphemes, separating a stem from its suffixes be it because local statistics are compatible with a break (in sublexical algorithms), or because it is economical to store the suffixes separately from their stems (in lexical algorithms). This behavior would be rewarded when the algorithm is evaluated on morpheme boundaries, but penalized when it is evaluated on word boundaries. More predictions on the performance of each specific algorithm are provided in the Appendix (see Section 6 below).

Given all of these reasons, we predict, in response to our first question, that languages that are morphologically more complex will result in lower segmentation scores than languages that are less complex, particularly when evaluated on the word level, but this may be mitigated when considering morphemes instead of words as a segmentation goal. Regarding our second question, we make no specific predictions, and approach it with an explorative and descriptive frame of mind.

\section{Experiment 1}

In this experiment, we study whether natural languages varying in morphological complexity differ in segmentability. We do so by assessing segmentability of two morphologically diverse languages, Japanese (Japonic) and Chintang (Sino-Tibetan) (see details on languages in Section 2.1.1).

Additionally, this study examines how considerable this effect is. To put the effect of language differences into context, we considered differences in performance across algorithms (see details on algorithms in Section 2.1.4), as well as differences in 
performance across evaluation levels.

Exploratory analyses assess whether included algorithms tend to segment morphemes or words by looking at how performance varies as a function of the level of linguistic representation on which segmentation is evaluated. If algorithms tend to segment morphemes, then performance will be better when evaluating on this level, but if they tend to segment words, performance will be better for orthographic words than for morphemes. We further report percentage of under- and oversegmentation on each level.

\subsection{Methods}

In this section, we detail several stages of analysis: language choice, corpus choice, corpus preparation, phonologization, segmentation, and evaluation. Corpus statistics, unsupervised word segmentation, and evaluation employed the WordSeg package (Bernard et al., 2018) ${ }^{3}$. The WordSeg package provides a collection of tools for text-based word segmentation. Statistical analyses were performed in R (R Core Team, 2013). More details can be found at https://osf.io/e8d2r/?view_only=f9d7b6a307734268bd8a515c55255b69. This OSF page contains scripts, results including segmentation performance and statistics, and supplementary material.

2.1.1 Languages. In this Experiment, corpora from two morphologically diverse languages are studied, Japanese and Chintang. Both languages were chosen on the basis of their typological characteristics and are part of the ACQDIV database, which contains longitudinal corpora of language acquisition for 10 maximally diverse languages (Moran, Schikowski, Pajović, Hysi \& Stoll, 2016, Stoll \& Bickel, 2013) 4

\footnotetext{
3 Available from https://github.com/bootphon/wordseg/.

4 To create this database, a new approach of sampling languages was introduced, called the Maximum-Diversity approach. More than 10 major typological variables that characterize inflectional marking (grammatical case, exponence, possessor agreement, inflectional compactness, syncretism, verb position, verb agreement, split ergativity of agreement markers, split ergativity of case, verbal synthesis, nominal synthesis) were considered (Stoll \& Bickel, 2013). Languages were sampled from the
} 
The main feature of interest in the present paper is their difference in the degree of synthesis, which allowed us to study the effect of morphological complexity. The degree of morphological synthesis was measured by looking for the maximally inflected verb and noun forms, and determining the number of grammatical and lexical categories (morphosyntactic features) encoded in that word form.

Chintang, a Sino-Tibetan language of the Kiranti branch (approx. 6000 speakers, Eastern Nepal), has higher verb and noun synthesis than Japanese (as just mentioned, measured in number of such categories expressed in the most complex word form; compare Bickel et al., 2007 for Chintang, and Kuno, 1973, Tsujimura, 1996 for Japanese), with up to 10 morphemes per word 5 . A good argument for the rich morphological system of the Chintang language is provided by Stoll et al. (2017), who point out there are 148 unique grammatical elements that can occur together with a verb stem in the corpus 5 Although some forms of verbs are rarely used, they constitute a part of the adult grammar and are eventually acquired by children. Here are two sample adult utterances from the Chintang corpus 78

two largest typological databases, WALS (Word Atlas of Language Structures) (Dryer \& Haspelmath, 2013) and AUTOTYP (Bickel et al., 2017. Nichols, Witzlack-Makarevich \& Bickel, 2013), resulting in 5 clusters of maximally diverse languages. Chintang and Japanese were in two different clusters.

${ }^{5}$ Phonological complexity (phonemic inventory and syllabic structure) is similar across the two languages (Bickel et al., 2007, Shibatani, 1990 Tsujimura, 1996).

${ }^{6}$ These elements include 120 grammatical markers and 28 secondary verb stems, called V2, that expand the lexical or grammatical meaning of the main verb.

${ }^{7}$ CLDLCh1R01S02.0044 and CLDLCh1R01S02.0057 respectively. In both examples, verbs are accompanied by four distinct functional morphemes.

${ }^{8}$ All abbreviations are based on the Leipzig Glossing rules. Specifically, for 1.a: DEM=demonstrative; ERG.A dig-3P-PRF-IND.PST-NMLZ=third person, perfect indicative past nominalized, ergative agent-like argument of canonical transitive verb. For 1.b: DEM.PROX=demonstrative proximal, $\mathrm{pst}=$ past $, \mathrm{ipfv}=\mathrm{imperfective}, \mathrm{emph}=\mathrm{emphatic}, \mathrm{ntvz}=$ nativizer, add=additive, fam=familiar . 
(1) a. ahã hun mi?muy namba-ya thok-u-ys-e-kha

no DEM a.little father.in.law-ERG.A dig-3P-PRF-IND.PST-NMLZ

'The father-in-law has dug it'

b. ba yay yug-a-yakt-a-kha ni ekchin-a

DEM.PROX ADD sit-PST-IPFV-PST-NMLZ EMPH little.while-NTVZ ekchin-a-kha

little.while-NTVZ-NMLZ

'He used to sit sometimes'

Japanese has moderate verb synthesis, expressing categories such as tense, voice, mood and polarity. A maximally inflected Japanese verb form would include 4-5 categories (Bickel \& Nichols, 2013b Hinds, 1986 Shibatani, 1990). Overall, Japanese has fewer forms both in its noun and verb paradigms compared to Chintang, and a smaller number of morphosyntactic features expressed especially in the verb. Here are two sample adult utterances from the Japanese corpus 9

(2) a. usagi-chan doko da

rabbit-FAM where be.PRES

'Where is Missy Rabbit?'

b. ocha mo doozo shi-te

tea too handing_over do-IMP

'Go ahead, make tea again'

To sum up, Chintang exhibits an extreme degree of morphological complexity. Thus, lower performance is predicted for Chintang, a morphologically more complex language, compared to Japanese, a morphologically less complex language.

${ }^{9}$ MYJCu44.1390521 and MYJCu832.1419814 respectively. 
2.1.2 Data. The Chintang recordings took place in a predefined week every month with several separated recordings amounting to approximately 4 hours per month involving 6 target children from 0;6-4;4 (Stoll et al., 2017). During the recordings, which were audiovisual, the children were mainly playing outside of their houses. Relatives, other children, and neighbors were part of their daily lives and this was captured in the recordings.

The Japanese data consist of 2 corpora, MiiPro (Miyata \& Nisisawa, 2009, 2010, Nisisawa \& Miyata, 2009, 2010) and Miyata (Miyata, 2004a, 2004b, 2004c). Recordings took place indoors, mostly at home and there was often just one caregiver conversing with the child. They contain data for 7 Japanese children aged 1;4-5;1 years old. For the MiiPro corpus, the recordings took place every week from $1 ; 2$ to $3 ; 0$ and later every 1 or 2 months, and lasted 70 minutes per session. For the Miyata corpus, recordings took place every week and lasted 40-60 minutes.

After data collection, both corpora were first transcribed orthographically, and then annotated morphologically. More information on the annotation process for Chintang can be found in Stoll and Schikowski (2020) and Gaenszle et al. (2005); see Miyata and Naka (2006) for information on annotation of the Japanese corpora. In the Chintang corpus, the transcription was done by native speakers and was susceptible to their impression of what an utterance was. This mainly corresponds to clauses marked by intonation. As for the Japanese corpus, utterances were defined on a syntactic basis, and only interjections like "un" or "hora" were adjoined based on prosodic information (Dr. Susanne Miyata, personal correspondence, 2021-04-16). Documentation suggests the data were transcribed based on the Wakachi format (Miyata \& Naka, 1998).

As for the morphological annotation, for Chintang, most of the corpus was hand-segmented and manually annotated for morphology and parts-of-speech by trained linguistic students under supervision by an expert in the language. A small part of the morphological annotation was generated automatically based on a morphological tagger (Ruzsics \& Samardzic, 2017, Samardzic, Schikowski \& Stoll, 2015). Japanese morphological tagging was done with the morphological tagger in CHILDES (JMOR, 
Miyata and Naka, 2014). For more information on the data, see also the ACQDIV manual (Schikowski, Moran \& Stoll, 2018).

2.1.3 Corpus preparation. Word segmentation algorithms usually take as input phonological, symbolic text-like representations such as phonemes or syllables, with few exceptions (e.g., Ludusan, Seidl, Dupoux and Cristia, 2015 and Roy and Pentland, 2002, who applied segmentation algorithms on raw speech data). There is evidence that even newborns have access to syllables (or vowels) as perceptual units (Jusczyk, Jusczyk, Kennedy, Schomberg \& Koenig, 1995), and that representation of phoneme sequences is available as early as by four months (Seidl, Cristià, Bernard \& Onishi, 2009). It is challenging to represent certain cues in text-like representations, such as coarticulation, which will therefore not be studied here. Here, we use phonemized representations as input (Moran \& Cysouw, 2018), without prosodic or coarticulation cues. Neither of the two languages exists in open source phonologization or text-to-speech programs, so we applied grapheme-to-phoneme rules to derive the phonological representation 10 (Moran \& Cysouw, 2018). We also cleaned the text removing punctuation and annotations.

Following Phillips and Pearl (2014b), we syllabified the corpora using the Maximal Onset Principle. According to this principle, the beginning of a syllable (onset) should be as large as legally possible (Bartlett, Kondrak \& Cherry, 2009, Kahn, 2015). We syllabified as follows, for each language separately. First, we made a list of vowels present in the corpus. Second, we made a list of all valid word-initial onsets, defined as all consonants up to the first vowel of the word or morpheme. Third, each utterance was processed from right to left until a vowel was found, at which point consonants to its left would be clustered to the maximally largest onset appearing in the list just

\footnotetext{
${ }^{10}$ Japanese had been transcribed in Latin script. The Chintang orthography is a relatively new orthography that represents language perfectly.

${ }^{11}$ All utterances containing "???" (which indicates incomprehensible speech or impossible morpheme annotation) were removed from both word- and morpheme-level analyses. We also removed utterances where one of the morphemes had been transcribed into an abstract, unpronounceable code (such as FS_N or kV), from both analyses.
} 
mentioned (Phillips \& Pearl, 2018). Notice that this procedure does not syllabify over morpheme or word boundaries (so boundaries cannot be re-syllabified or obscured).

Both corpora are larger than those frequently used for modeling studies (e.g., Saksida et al., 2017, 10,000 words; Phillips and Pearl, 2014b, 30,000 utterances). This allowed us to further divide each corpus into ten equal subsets, based on their length measured in number of utterances. The subsets gave us an estimate of variation in the properties of the corpora and of the algorithms, and informed us on the amount of data required for each algorithm to converge. As we show in the Results (Section 2.2), performance for the whole corpus lies within the variance of results for the 10 subsets, which guarantees that the data used in this paper were more than enough for our algorithms to converge successfully. This division further allowed us to employ the statistical analyses described in Section 2.1.5.

After pre-processing, the entire Japanese corpus was 84,518 utterances long and had 155,805 word tokens. The Chintang corpus was 152,571 utterances long and had 426,288 word tokens. Table 1 gives properties of the subsets after pre-processing. Right before segmentation, within-utterance word boundaries were removed from the corpora, and only utterance boundaries remained. 


\begin{tabular}{|c|c|c|}
\hline Mean subset stats & Chintang & Japanese \\
\hline $\mathrm{Nb}$ utt & 15,257 & 8,451 \\
\hline Nb wtokens & $42,628(1,371)$ & $15,579(1,569)$ \\
\hline Nb wtypes & $8,194(690)$ & $1,634(361)$ \\
\hline Nb whapaxes & $5,180(514)$ & $812(206)$ \\
\hline $\mathrm{Nb}$ wtokens/utt & $2.79(0.09)$ & $1.84(0.19)$ \\
\hline $\mathrm{Nb}$ wtypes/utt & $0.54(0.05)$ & $0.19(0.04)$ \\
\hline $\mathrm{Nb} \mathrm{m} / \mathrm{utt}$ & $4.72(0.29)$ & $1.96(0.20)$ \\
\hline $\mathrm{Nb}$ syll/utt & $5.62(0.25)$ & $3.38(0.41)$ \\
\hline $\mathrm{Nb}$ phon/utt & $11.87(0.60)$ & $6.58(0.83)$ \\
\hline Nb mtokens/wtokens & $1.69(0.07)$ & $1.07(0.03)$ \\
\hline $\mathrm{Nb}$ mtypes/wtypes & $0.25(0.02)$ & $0.84(0.03)$ \\
\hline $\mathrm{Nb}$ syll/w & $2.01(0.05)$ & $1.83(0.07)$ \\
\hline $\mathrm{Nb}$ phon/w & $4.25(0.12)$ & $3.56(0.15)$ \\
\hline $\mathrm{Nb}$ syll/m & $1.24(0.03)$ & $1.70(0.04)$ \\
\hline $\mathrm{Nb}$ phon/m & $2.56(0.05)$ & $3.33(0.09)$ \\
\hline
\end{tabular}

Table 1

Corpus features: Means (and standard deviation) across the ten Chintang and Japanese subsets (see main text for explanation). "Nb" stands for number, "utt" stands for utterance. "wtokens", "wtypes", "whapaxes" stand for word tokens, word types and word hapaxes respectively. "m", "syll" and "phon" stand for morphemes, syllables and phonemes, respectively. 
Regarding the use of orthographic words, it was preferable over other definitions of wordhood for several reasons. First, it allows comparison with previous computational work. Second, it is unclear that it is much worse or much better than alternative definitions. Phonological and morphosyntactic criteria for word segmentation are also problematic and cannot decide controversial cases: Phonological words may not be consistent within and across languages (Schiering, Bickel \& Hildebrandt, 2010) and they often fail to coincide with morphosyntactic words (Dixon \& Aikhenvald, 2002). For this, and as previous segmentation studies did, we used the existing orthographic word boundaries for our word level.

2.1.4 Algorithms. A brief, cognitively-focused introduction to the algorithms follows. For more technical details, please refer to the WordSeg documentation (wordseg.readthedocs.io Bernard et al., 2018) and the work cited for each algorithm. We used open-source models from the WordSeg package. As the authors of the package explain (Bernard et al., 2018, p. 266), models were selected based on (a) code availability, and (b) dimensions of variation found in a systematic review of previous computational work. Thus, it is not an exhaustive set. Nevertheless, the models we use in the present paper vary largely in complexity and cognitive assumptions, in an effort to include main strategies previously considered in the field of language acquisition (e.g., Daland and Pierrehumbert, 2011; Goldwater, Griffiths and Johnson, 2009 Saffran, Aslin and Newport, 1996 - see also Section 1.1). The only exception are the 'baseline' models, which were included in WordSeg because it was relevant to compare performance against a baseline (but see Section 1.1, where we argue that the baselines used in this paper may also be plausible strategies for segmentation).

The first algorithm, a member of the Adaptor Grammar family, adopts a lexical approach (Goldwater et al., 2009, M. Johnson \& Demuth, 2010, M. Johnson, Griffiths \& Goldwater, 2007). The Adaptor Grammar (AG) is a generalized version of probabilistic context-free grammars (PCFG, M. Johnson et al., 2007). We use a very simple hierarchical grammar with only a few rules: Sentences are composed of one or more reusable words (or morphemes), and words/morphemes are composed of one or more 
phonemes. Each utterance is parsed as a sequence of words/morphemes, each word/morpheme is composed by phonemes, and a given word/morpheme of this sequence would be generated either by choosing an existing form from a lexicon based on previous occurrences, or by considering it as a novel item and inserting its phonemic form in the lexicon. The PCFG regenerates the corpus by repeatedly applying this grammar, which is a set of rewrite rules with assigned probabilities. The rules fit the corpus based on how elements have already been written in the past, according to the Pitman-Yor stochastic process, which favors the reuse of frequently occurring rules (M. Johnson et al., 2007). Pitman-Yor yields word frequency distributions that follow a power-law distribution, a widely attested pattern in natural languages known as Zipf's law (Zipf, 1935). Zipf's Law states that in a large corpus, the frequency of any word is inversely proportional to its rank in the frequency table. AG would thus tend to create a lexicon of moderate size comprised mostly of short words (Perfors \& Navarro, 2012).

DiBS (Daland, 2009) performs segmentation using phone bigram probabilities. As a consequence, this algorithm requires that the input be coded as a sequence of phonemes. The intuition behind this algorithm is that certain sound sequences almost never occur within words (or morphemes), so if observed they probably indicate a word boundary. For instance, when [pd] occurs in English, the probability that there is a word boundary is very high: $\operatorname{Pr}(\# \mid p d) \approx 1$.

The unsupervised version (phrasal DiBS) treats utterance edges as a proxy for word edges, assuming that phoneme sequences frequently spanning utterance boundaries likely also span word boundaries. The algorithm estimates the necessary parameters from data using the Formula 1, where $f\left(x \#_{u} y\right)$ is the number of [xy] sequences with an uttterance boundary in the middle and $f(x y)$ is the number of [xy] sequences in any position, and where $\mathrm{x}$ is one phoneme, $\mathrm{y}$ is another phoneme, $\left(\#_{u}\right)$ is an utterance boundary and $\left(\#_{w}\right)$ is a word boundary:

$$
\mathrm{p}\left(\#_{w} \mid \mathrm{xy}\right) \approx \frac{\mathrm{f}(\mathrm{x \#}, \mathrm{y})}{\mathrm{f}(\mathrm{xy})}
$$

When $p\left(\#_{w} \mid x y\right)$ is higher than a threshold parameter, the system breaks the sequence 
by positing a word boundary. This threshold parameter is estimated with Formula 2

$$
\frac{\mathrm{Nw}-\mathrm{Nu}}{\mathrm{Np}-\mathrm{Nu}}
$$

where the total number of words is $N w$, the number of phonemes is $N p$ and the number of utterances is $N u$.

The TP family is a group of algorithms that assume that unit-internal pairs of syllables tend to co-occur more frequently than the same pairs across unit boundaries (whereby unit could be word or morpheme, extrapolating from the definition on page 610 of Saffran, Newport and Aslin, 1996), thus the transitional probability between adjacent syllables is higher word-internally than at word boundaries (cf. Gervain and Erra, 2012 for evidence that this may not be the case). The basic minimal units are syllables and not phonemes, unlike in the other two algorithms. Forward transitional probabilities (FTP) are defined as:

$$
\operatorname{FTP}(\mathrm{AB})=\frac{\mathrm{f}(\mathrm{AB})}{\mathrm{f}(\mathrm{A})}
$$

where $f(A B)$ is the frequency of a syllabic sequence $A B$ and $f(A)$ is the frequency of the syllable A. Backward TP (BTP) is similar, except that the denominator is the frequency of the second syllable instead.

$$
\mathrm{BTP}(\mathrm{AB})=\frac{\mathrm{f}(\mathrm{AB})}{\mathrm{f}(\mathrm{B})}
$$

Also, algorithms in the TP family require another parameter, namely the threshold used to decide whether to add a word (or morpheme) boundary or not. One possibility is to use TP with a Relative threshold, i.e. BTPr and FTPr, which leads to placing a word/morpheme boundary wherever the TP value of a syllable pair is lower than the TP of the neighboring syllable pairs, as follows. Given a syllable sequence $(W A B Y)$ where $W, A, B, Y$ stand for syllables, a break will be posited between $\mathrm{A}$ and $\mathrm{B}$ if $T P(W A)>T P(A B)$ and $T P(B Y)>T P(A B)$.

Another possibility is to use TP with an Absolute threshold (BTPa and FTPa), which would posit boundaries using a threshold, that is the sum TP value of all syllable pairs over the number of different syllable pairs. For example, given a corpus consisting 
of a syllable sequence $(W A B Y)$ where $W, A, B, Y$ stand for syllables, the absolute threshold is $T P a=\frac{T P(W A)+T P(A B)+T P(B Y)}{3}$. A break will be then posited between A and $\mathrm{B}$ if $T P(A B)<T P a$.

Note that there can be large differences in performance within the same algorithm depending on the parameters used (e.g., Gervain and Erra, 2012; Saksida et al., 2017). For example, Saksida et al. (2017) documented that different measures such as forward transitional probabilities, backward transitional probabilities and especially the threshold parameter (absolute, relative) can affect the results of word segmentation.

Finally, we applied two segmentation baselines. The baselines capture simple segmentation strategies. The baseline called $\mathbf{S y l l}=\mathbf{U n i t}$ uses $p=1$, where $p$ is the probability of adding a boundary after a syllable, meaning that every single syllable is followed by a boundary. As a result, it treats every syllable as a word (or morpheme). The baseline $\mathbf{U t t}=\mathbf{U n i t}$ labels only utterance boundaries as word (or morpheme) boundaries ( $p=0$, meaning that no boundaries are inserted within utterances).

2.1.5 Evaluation. The output of each algorithm is evaluated using word or morpheme token F-scores, derived from Precision and Recall, as standard metrics for segmentation algorithm evaluation (Phillips \& Pearl, 2015). In this paper we use the more intuitive terms Model Token Accuracy to describe Precision, and Corpus Token Accuracy to describe Recall. Model Token Accuracy amounts to how many words/morphemes in the set of those segmented by the algorithm are correct. Corpus Token Accuracy checks how many words/morphemes in the set of those existing in the 
original gold corpus were correctly segmented by the algorithm.

The Token F-score balances how accurate and complete the set of identified word/morpheme tokens is (Phillips \& Pearl, 2015). It is the harmonic mean of Model and Corpus Token Accuracy, as shown in Formula 7.

$$
\text { F-score }=\frac{2 * \text { Model Token Accuracy } * \text { Corpus Token Accuracy }}{\text { Model Token Accuracy }+ \text { Corpus Token Accuracy }}
$$

As mentioned in Section 2.1.3, we separated each language corpus into 10 different subsets. We evaluated segmentation both of the entire corpus, and of each one of the subsets. The separation into 10 subsets was motivated by several reasons. It allowed us to employ regression analyses as described in the next paragraph, and it provided an estimation of variability within each corpus. This separation was also informative of the amount of data necessary for these segmentation strategies to produce fairly stable results (see Section 2.1.3). Note incidentally that we were in a better position to do this separation than much previous work, since the Chintang and Japanese corpora at our disposal were large enough to allow measuring the segmentation effects in separate subsets.

Finally, a linear regression predicting F-scores from language, level, algorithm, with a random effect of different subsets assessed the effect and significance for each one of the three factors and their interactions. ${ }^{13}$ Given our predictions in Section 1.4 , we expected the language coefficient slope to be positive for Japanese. We compared the

12 Model and Corpus Token Accuracy are defined based on true and false positives and negatives, as follows (note that the two formulas only differ on the second term of the denominator):

$$
\begin{aligned}
\text { Model Token Accuracy } & =\frac{\text { true positives }}{\text { true positives }+ \text { false positives }} \\
\text { Corpus Token Accuracy } & =\frac{\text { true positives }}{\text { true positives }+ \text { false negatives }}
\end{aligned}
$$

True positives are the words/morphemes segmented by the algorithm which are indeed found in the input corpus. False positives are the words/morphemes segmented by the algorithm which are actually not in the input corpus. False negatives are words/morphemes in the input corpus that were not in fact segmented by the algorithm.

13 The function was: $\operatorname{lm}$ (token fscores $\sim$ language $*$ level $*$ algorithm $+(1 /$ subset $)$, data $).$ 
size of this language effect to the coefficients of algorithm and level. We further tested the effects through an analysis of variance (ANOVA type III) for all factors and interactions on a linear regression with token F-scores as the dependent variable.

\section{$2.2 \quad$ Results}

Even though all analyses are based on segmentation F-scores, we start by presenting an illustration of Model and Corpus Token Accuracy for each subset of the corpora (Fig. 1). The point of this figure is to demonstrate that Model and Corpus Token Accuracy are correlated to a considerable extent. The correlation between Model and Corpus Token Accuracy emerges because there is no trade off between false negative and false positive results; when segmenting text, a given parse results in neither or both kinds of errors. This is because if a boundary is posited, then if this boundary is correct, it will increase both Model Accuracy and Corpus Accuracy. If the boundary is incorrect, it will reduce both Model Accuracy and Corpus Accuracy.

Since Model and Corpus Token Accuracy are highly correlated, we focus on the more commonly reported Token F-scores. Fig. 2 shows for each language, F-score results for the entire corpus, as well as for each of the 10 subsets. Results for the entire corpus were nearly always contained in the range of variation of the subsets. Similarly to what we found, Bernard et al. (2018) documented that variation in corpus size beyond the first $5 \mathrm{k}$ utterances seems to play a negligible role in performance of these segmentation systems, as replicated here. Fig. 2 suggests that there were strong interactions between the three factors of interest (language, algorithm, and level), which are tested statistically in the next section. All F-scores are presented numerically in the online supplementary materia ${ }^{14}$

Before moving on, it is important to discuss what may constitute good performance, given that the goal here is to inform our understanding of infant language acquisition. We are looking at segmentation using purely distributional cues, and thus

\footnotetext{
${ }^{14}$ Link: https://osf.io/e8d2r/?view_only=f9d7b6a307734268bd8a515c55255b69, folder 'results', file 'results.csv'
} 


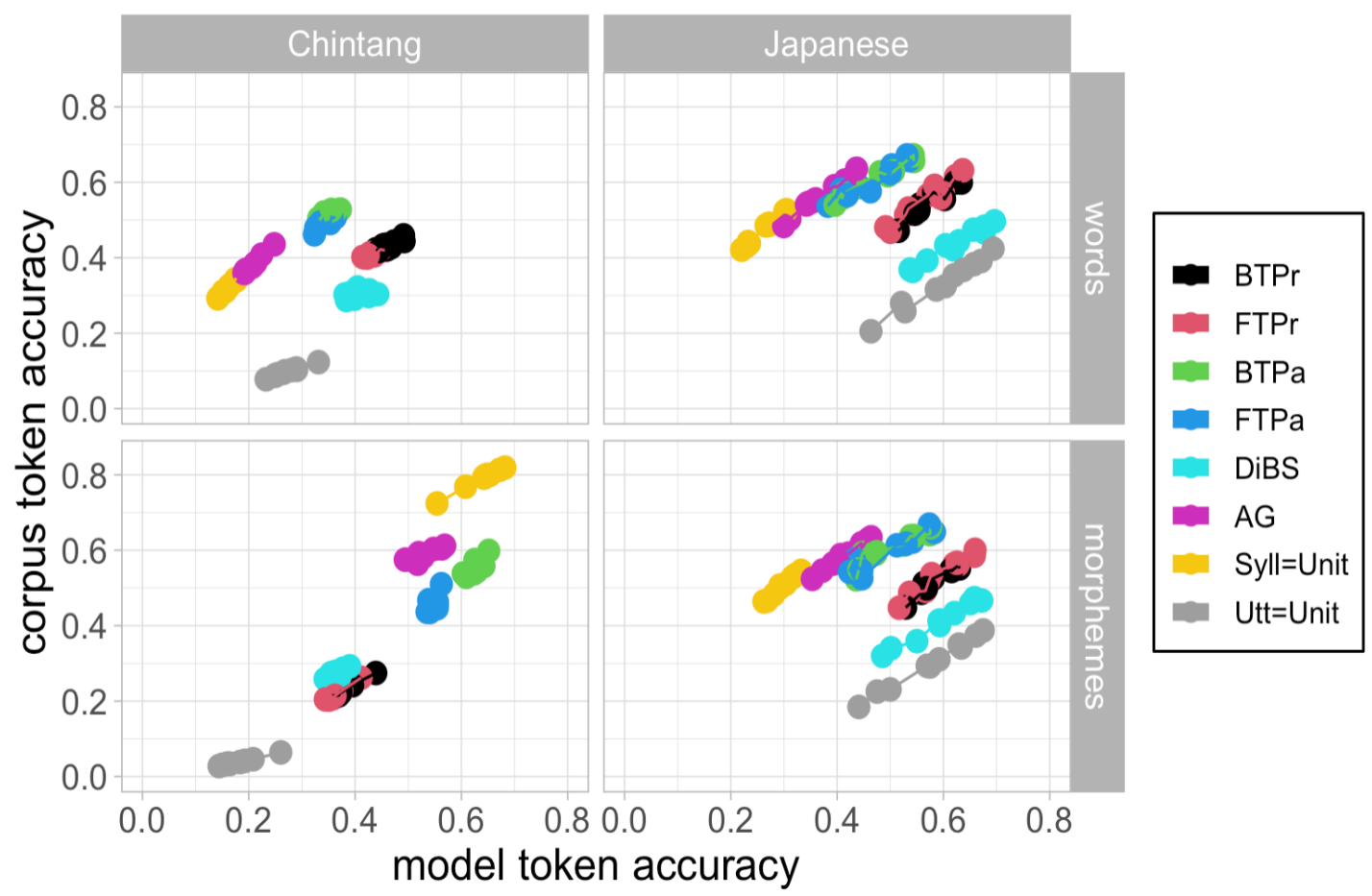

Figure 1. Model and Corpus Token Accuracy across languages and algorithms for each evaluation level. Algorithms are marked by color. BTPr, FTPr, BTPa or FTPa indicate the segmentation result for one of the different versions of TP. AG are the results of the unigram Adaptor Grammar. Syll=Unit and $\mathrm{Utt}=$ Unit are the results of the baselines. Each dot indicates the result for one of the ten subsets of a given corpus.

it is not reasonable to expect that segmentation would be perfect. We know that adults can use other sources of information, including meaning, grammatical structures, and context (Davis, Marslen-Wilson \& Gaskell, 2002; Gow \& Gordon, 1995). Additionally, we are testing the algorithms exhaustively, on every word in every sentence, in a way that is completely unforgiving, whereas all work on humans (both infants and adults) relies on partially sampling from that experience during test. Finally, even in adulthood, we know that segmentation errors can occur, as in the recurrent mis-segmentation of Bob Dylan's song "the ants are my friends" (instead of "the answer, my friends").

2.2.1 Regression on token F-Scores. A regression predicting F-scores from language, level, algorithm, and their interactions accounted for most variance in the data, $R^{2}=.93(F(31,288)=134.95, p<.001) .15$ Even though the presence of

\footnotetext{
15 The function was: $\operatorname{lm}($ token fscores $\sim$ language $*$ level $*$ algorithm $+(1 /$ subset $)$, data $)$. Token F-scores are the F-scores to be predicted by language, level, and algorithm as fixed effects, and subset as random
} 


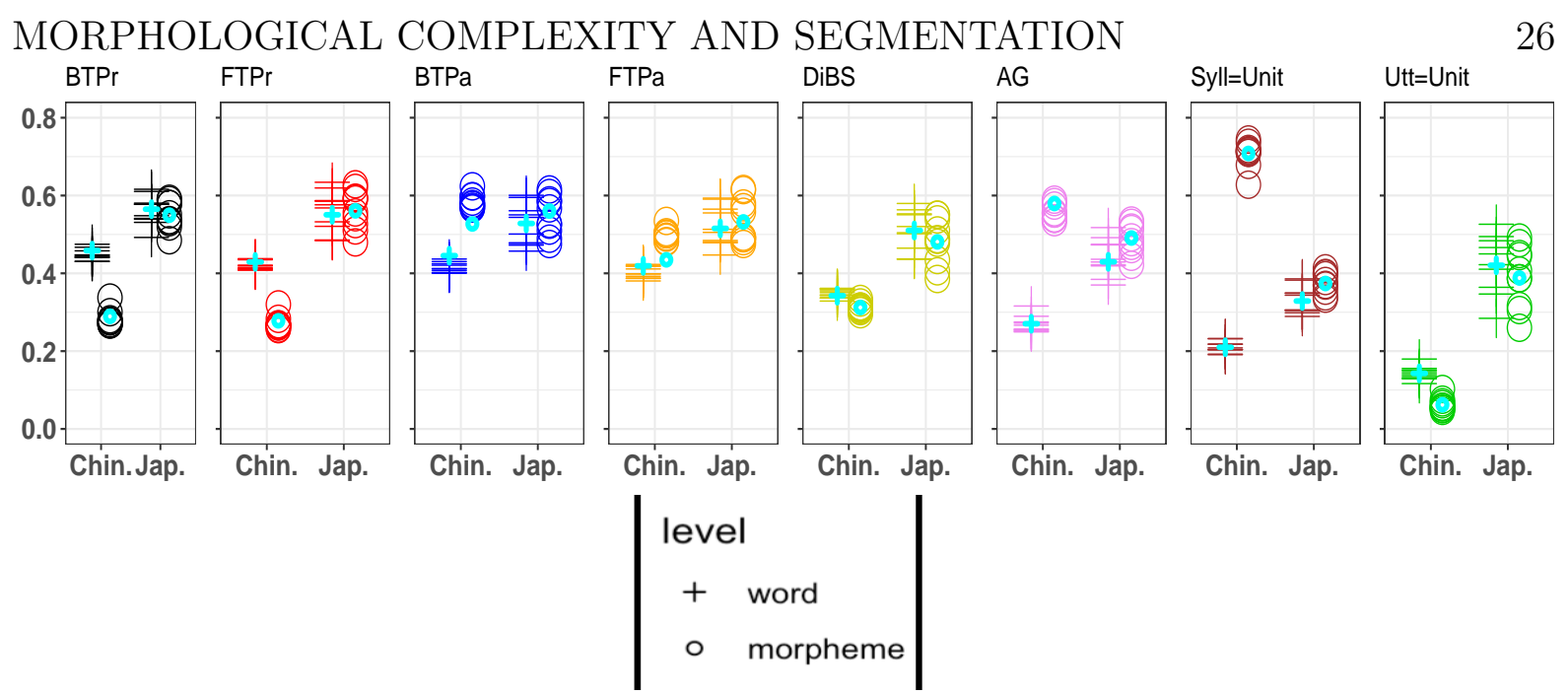

Figure 2. Token F-scores (y axis) across language (Chintang, Japanese) and level (words, morphemes). Evaluation levels are marked by shape. Each circle or cross shows the results for a subset. Each cyan circle or cross shows the result for a corpus segmented as a whole. Color reflects algorithms (which also group the data into boxes, see main title of each box). BTPr, FTPr, BTPa and FTPa indicate the different versions of Transitional Probabilities. AG is the unigram Adaptor Grammar. Syll=Unit and Utt=Unit are the baselines. Chin. stands for Chintang and Jap. stands for Japanese.

significant interactions precluded a direct interpretation of the main effects, the regression confirmed an advantage for Japanese, with a positive coefficient estimating the language effect (0.11). This bears on our first research question, on whether there are language effects. In response to our second research goal, language effects appear to be relatively small, since the F-value for language is about half that of level and algorithm. Thus, the answer to our second research question is that language differences related to morphological complexity are small, when compared against effects due to which algorithm is used for segmentation and whether morphemes or words are evaluated. Full regression results are provided in the online supplementary material (https://osf.io/e8d2r/?view_only=f9d7b6a307734268bd8a515c55255b69 supplementary_material.pdf, p. 3).

2.2.2 Over-, under- and missegmentation. Given that this is the first study to look at potential language differences due to morphological complexity, we sought to inform future work through exploratory descriptive analyses by describing factor. The data frame contains 320 observations (2 languages $\mathrm{x} 2$ levels $\mathrm{x} 10$ subsets $\mathrm{x} 8$ algorithms). 
potential differences in the pattern of over-, under-, and mis-segmentation as a function of language, level, and algorithm type. In the current study, we operationalize oversegmentation as the splitting up of a unit in one or more sub-parts (regardless of whether these are reasonable smaller units at a different level of analysis). We consider undersegmentation the clustering together of two or more units. All other differences from the gold segmentation were labeled missegmentation. The following example illustrates how they were implemented: If the input sentence "the dog ate the other dog" is returned as "thedog at ethe other $d$ og", then the word-level score will be $1 / 6$ correct segmentation, $1 / 6$ over-segmentation ("d og"), 2/6 under-segmentation (one for each input word, "the" -the first token- and "dog"), and 2/6 mis-segmentation ("at ethe").

Over-, under- and mis- segmentation are measured on their corresponding evaluation unit. For example, on the morpheme level, if the Japanese sentence 'kore nani da', containing three morphemes, was segmented as 'ko re nani da', 1 out of 3 morphemes would be considered oversegmented. The prevalence of over-, under- and missegmentation as a function of language, level, and algorithm are reported in Table 2 and discussed next.16

\footnotetext{
16 Additionally, a breakdown of segmentation performance as a function of part-of-speech and algorithm is provided in the online supplementary material (supplementary_material.pdf, p. 4).
} 
Table 2

\begin{tabular}{|c|c|c|c|c|c|c|c|c|c|c|c|c|c|c|c|c|}
\hline algo & \multicolumn{3}{|c|}{ Chin. words } & \multicolumn{3}{c|}{ Chin. morph. } & \multicolumn{3}{|c|}{ Jap. words } & \multicolumn{3}{|c|}{ Jap. morph. } \\
\hline & ov & un & mis & cor & ov & un & mis & cor & ov & un & mis & cor & ov & un & mis & cor \\
\hline AG & 54 & 0 & 7 & 38 & 14 & 10 & 15 & 61 & 39 & 3 & 3 & 55 & 30 & 3 & 7 & 59 \\
BTPa & 28 & 9 & 11 & 53 & 4 & 47 & 2 & 47 & 23 & 13 & 2 & 61 & 19 & 16 & 3 & 62 \\
FTPa & 27 & 11 & 13 & 49 & 5 & 53 & 4 & 38 & 24 & 13 & 3 & 60 & 19 & 17 & 5 & 58 \\
BTPr & 7 & 20 & 29 & 45 & 2 & 70 & 5 & 23 & 9 & 25 & 11 & 55 & 6 & 31 & 11 & 52 \\
FTPr & 7 & 20 & 31 & 42 & 1 & 70 & 7 & 22 & 9 & 21 & 15 & 55 & 6 & 27 & 13 & 53 \\
DiBS & 6 & 47 & 17 & 30 & 7 & 47 & 18 & 27 & 2 & 49 & 6 & 43 & 2 & 51 & 7 & 41 \\
\hline Syll=Unit & 68 & 0 & 0 & 32 & 21 & 0 & 0 & 79 & 53 & 0 & 0 & 47 & 49 & 0 & 0 & 51 \\
Utt=Unit & 0 & 90 & 0 & 10 & 0 & 96 & 0 & 4 & 0 & 68 & 0 & 32 & 0 & 71 & 0 & 29 \\
\hline
\end{tabular}

Percentage of oversegmented, undersegmented, missegmented and correctly segmented tokens for each algorithm, level, and language. "algo" stands for algorithm, "morph." for morphemes, "Chin." for Chintang and "Jap." for Japanese. Also, "ov" stands for oversegmentation, "un" for undersegmentation, "mis" for missegmentation and "cor" for correctly segmented.

Comparison across levels (e.g., Chintang words versus Chintang morphemes, Japanese words versus Japanese morphemes) reveals that oversegmentation rates were higher for both languages when evaluating on words than when evaluating on morphemes. This is consistent with an interpretation whereby algorithms oversegment when evaluated at the word level because they break off affixes. Especially AG, the lexical algorithm, was more likely to break apart morphological subparts at the word level than the sublexical DiBS and TP (see Appendix, Section 6 for details on the performance of each algorithm).

Additionally, the differences between morpheme-level and word-level evaluation is stronger for Chintang than Japanese, again consistent with that interpretation, as the risk of breaking off affixes will be higher in the former, more complex language. Notice, however, that while oversegmentation was substantially reduced when evaluating on morphemes, it did not disappear. This may mean that algorithms are finding breaks and/or subsections even within affixes that linguists coded as being holistic units. This 
may be simply segmentation errors, or it could reveal patterns for which expert linguists (and/or infants) may provide some meaningful interpretation (as in series like "glitter, glimmer, glisten" - linguists typically do not treat "gl" as an affix, but can interpret it as a common feature of these words).

\subsection{Discussion}

In Experiment 1, a set of segmentation algorithms was applied to corpora of two morphologically diverse languages, and the output was assessed against gold standard segmentation at the word and morpheme level. Given the details of Chintang morphology, it was hypothesized that such a rich morphology must pose significant problems for the uninformed learner who is trying to segment the input, but these problems may be mitigated if we consider morphemes instead of words as a segmentation goal.

Regarding our first research goal, results summarized thus far support the prediction that languages varying in morphological complexity might vary in segmentability, although other aspects of these results suggest that the answer is not simple: The strength of the language effect varied across algorithms, and was even reversed in some conditions. In fact, turning to our second research goal, the language effect was considerably smaller than the effects found for the other two factors we studied, namely level and algorithm. In other words, differences within-language across algorithms or segmentation levels were more important than those between languages. We also found interactions between language, level, and algorithm, to which we turn by considering the results within each algorithm next (see also the Appendix for an interpretation).

The results of the lexical algorithm, AG, matched our predictions well. In AG, we observed higher performance for Japanese than Chintang with words as the gold standard, but this difference was reversed with morphemes. This observation is consistent with the proposal that AG, and probably lexical algorithms in general, are ideal to recover recombinable units - i.e., morphemes. The fact that AG performed 
better when evaluated on morphemes than words means that, in order to build a lexicon, it tends to segment minimal recombinable units of the size of morphemes, including affixes. So, languages like Chintang, with many affixes, may be segmented better by this algorithm at the morpheme level than simpler languages such as Japanese. Thus, lexical algorithms might work well for languages like Chintang, improving performance on the morpheme level.

Turning to the sublexical algorithms, the four versions of the TP algorithm (BTPa, FTPa, BTPr, FTPr) yielded divergent patterns. This is in accordance with previous findings, where notable differences in performance were found depending on the parameters used (e.g., Saksida et al., 2017). TP was also sensitive to morpheme boundaries - it seems that when morphemes are combined with different stems, the transitional probabilities between them are low, and they may be segmented by this algorithm. Last, even though DiBS is a phonotactic-based algorithm, it interacted with language and was affected by language differences.

In further analyses, we attempted to quantitatively evaluate segmentation across levels when considering the languages (Chintang, Japanese) and models of our study, by describing the proportion of tokens that were under- or over-segmented. Our results converged with previous qualitative reports: it has been argued that lower segmentation performance for some morphologically complex languages could arise from oversegmentation when evaluating on words because segmentation algorithms may break off affixes (Gervain \& Erra, 2012; M. Johnson, 2008). For instance, Gervain and Erra (2012) inspected their results applying algorithms from the TP family on a Hungarian corpus, and noticed that some of the segmented material formed real morphemes, which is interesting given that TP-based algorithms are not informed about lexical and morphological composition. Similarly, Fourtassi et al. (2013) segmented an English and a Japanese corpus using a lexical algorithm. Qualitative inspection showed that the algorithms broke off morphological affixes, with more oversegmentation cases for Japanese than English. We can now precisely pinpoint these effects quantitatively: We confirm that the percentage of items that are over-segmented 
is lower when evaluating on morphemes than words, and that some of the apparent over-segmentation of words is due to decomposing words into morphological units. We also add that this effect is more marked in the lexically-driven AG algorithm than in algorithms from the sublexical TP family.

There are some limitations of this experiment that we would like to acknowledge. First, the research was conducted using transcriptions of speech spoken around (and not only to) children varying in age, from a few months to five years old, with the Japanese children being in general older than the Chintang children. Second, two different datasets compose the ACQDIV Japanese corpus, as mentioned in Section 2.1.2, which might be the cause for the ostensibly more variable results of the Japanese subsets.

Moreover, since Chintang is spoken in a multilingual setting, annotators transcribed all speech including non-Chintang words, either because they are recent loanwords or because of code-switching into Nepali. Most Chintang speakers are bilingual in the morphologically simpler Nepali and children encounter Nepali from early on (Stoll, Zakharko, Moran, Schikowski \& Bickel, 2015). In fact, $36 \%$ of the Chintang utterances had non-Chintang single- or multi-word insertions. For our analyses, we chose to report on the results for the whole corpus, because children born into this community do not come with information about which words are loanwords or code-switched. However, we also segmented a version of the corpus consisting of only all-Chintang utterances, where utterances with non-Chintang insertions had been removed. The performance usually increased by .01-.06 in Token F-scores for the all-Chintang corpus, and did not alter our conclusions above (see Loukatou, Stoll and Cristia, 2018 for a similar analysis).

All of these limitations are conceptually related: We compared two languages, represented by two naturalistic corpora, so we cannot be certain that the differences we observe are due to the specific factor that led us to choose these two languages or to any extraneous factor in the corpora and/or to other uncontrolled characteristics of the languages. We considered extending this approach to other natural child-centered corpora, for instance by looking at 10-20 corpora of languages varying in morphological 
complexity. This turned out not to be feasible, since morphological segmentation is typically not available in child-centered corpora. Moreover, there would always have been the possibility that uncontrolled differences caused, or obscured, any result that we were to find.

To study the effects of morphological complexity on segmentability in a more controlled fashion, we created artificial languages in two follow-up experiments. In Experiment 2 (Section 3) we segment five artificial languages varying in their number of affixes, and in Experiment 3 (Section 4) we segment three artificial languages varying in the distribution of their affix number.

\section{Experiment 2: Artificial languages varying in the number of affixes}

In Experiment 1, we looked at Chintang and Japanese through naturalistic corpora that may differ across uncontrolled factors. For example, Chintang parents could by chance produce longer utterances, and this would affect our results. Since it is impossible to control for this in naturalistic corpora, we performed additional experiments with artificial languages. Artificial languages allow us to study specific properties of languages and their effect under tightly controlled conditions.

In Experiment 2, we study whether languages varying in morphological complexity differ in segmentability by assessing segmentability of five morphologically diverse artificial languages, which exhibit a gradual range of morphological complexity. We make sure that the number of words per sentence are matched across corpora, and the languages only differ on this specific aspect of morphological complexity.

We focus on morphological synthesis, keeping all other variables stable. In order to study the effects of morphological synthesis on segmentability, we track changes in segmentation while modifying the ratio of morphemes per word. In Experiment 1, the mean number of morphemes per word was about 1.69 for Chintang and 1.07 for Japanese. In Experiment 2, we increase the variability of this feature, ranging from 0 to 4 affixes accompanying the stem.

The same questions asked in Experiment 1 are revisited in this controlled 
experiment. First, do languages varying in morphological complexity differ in segmentability? Based on the key predictions above, languages with a smaller number of morphemes within words should be easier to segment than languages where words have multiple morphemes. Also, based on the key predictions above, lexical and sublexical algorithms should yield lower word segmentation scores for more complex languages, as they are more likely to break up the stream at morpheme boundaries.

Second, how large is this effect, compared to differences across algorithms and level? We inquire whether performance varies as a function of algorithm (the specific algorithm employed during segmentation) and the level of linguistic representation on which segmentation is evaluated (words or morphemes). We concluded in Experiment 1 that morphology-related differences across languages were relatively small, but this conclusion could be curtailed by the fact that the range of variation covered with these two natural languages may be small. Experiment 2 allows us to better measure these effects by studying them while controlling all other sources of variation, and substantially increasing the range of linguistic variation covered.

\subsection{Methods}

3.1.1 Languages. Languages were created using a script in R. ${ }^{17}$ First, a set of consonant-vowel syllables were composed through every combination of 11 consonants and 5 vowels. We then composed a lexicon of 1,000 words. Function words constituted $1 \%$ of this lexicon, and they were always one syllable in length. The rest of the lexicon were content word stems, which varied in length between 1 and 4 syllables. Content words were randomly split into two classes, A and B (which may be thought of as nouns and verbs), and which selected affixes from two different paradigms. All of these aspects were fixed across languages.

Languages varying in complexity thus differed only on the next step. The base language (0) had no affixes; the next language (1) had one affix per content word (with

\footnotetext{
17 The script can be found at https://osf.io/e8d2r/?view_only=f9d7b6a307734268bd8a515c55255b69 folder 'scripts', file 'create-lang_exp2-3.R' and folder 'data', 'artificial_languages.zip'.
} 
different affixes for class A and B stems); and so on, for up to 4 affixes (4). All affixes were one syllable long. Table 3 shows some basic statistics of these languages.

\begin{tabular}{|c|r|r|r|r|r|}
\hline Mean subset stats & 0 & 1 & 2 & 3 & 4 \\
\hline Nb utt & 5,000 & 5,000 & 5,000 & 5,000 & 5,000 \\
\hline Nb wtokens & $12,528(55)$ & $12,528(55)$ & $12,528(55)$ & $12,528(55)$ & $12,528(55)$ \\
Nb wtypes & $791(8)$ & $4,695(36)$ & $7,125(31)$ & $7,482(23)$ & $7,520(21)$ \\
Nb whapaxes & $1.5(0.71)$ & $2,807(43)$ & $6,733(38)$ & $7,428(26)$ & $7,506(20)$ \\
Nb phtokens & $50,535(460)$ & $65,515(376)$ & $80,581(525)$ & $95,570(518)$ & $110,556(490)$ \\
Nb mortokens & $12,528(55)$ & $20,044(75)$ & $27,560(93)$ & $35,073(114)$ & $42,582(129)$ \\
\hline
\end{tabular}

Table 3

Means (and standard deviation) of corpus features across the ten subsets of artificial languages 0, 1, 2, 3, and 4 (see main text for explanation). "Nb" stands for number, "utt" for utterance. "wtokens", "wtypes", "whapaxes" stand for word tokens, word types and word hapaxes. "phtokens" stands for phoneme tokens and "mortokens" stands for morpheme tokens.

The final step was also in common across languages, and consisted in creating a corpus of 5,000 sentences that were between 1 and 4 words in length. Previous methodological work suggests algorithms' performance is stable by about 5,000 sentences (Bernard et al., 2018). Sentence lengths of 1-4 seem reasonable for child-directed speech, according to previous descriptive studies (Loukatou, Le Normand \& Cristia, 2019). Sentences one word in length had only a stem (and, for more complex languages, its affixes); sentences with two words had a function word and a stem (and affixes); three-word sentences had a function word and two stems (and their affixes); and four-word sentences had a function word, a stem (and its affixes), a function word, and a stem (and its affixes). For clarity in the code, each sentence sampled from the lexicon for each language separately.

To make this more concrete, here is the first sentence in the five languages' corpora in one run, containing always three words (a function word followed by two stems with their eventual affixes, depending on the language); words are separated by spaces, morphemes by dashes: 
- 0: "pi rotu rodezira"

- 1: "yu so-se qofeharu-se"

- 2: "tu yosoreda-ga-yi foyo-gi-su"

- 3: "pi ruza-to-re-pu gori-di-re-ra"

- 4: "fe zi-pa-yo-ye-gi ho-fa-ge-ye-te"

And the following are sample sentences containing the stem "rodezira", which was one of the stems in the lexicon in that run, appearing in sentences of the same word length across the five languages:

- 0: "pi rotu rodezira" (3 words, 3 morphemes)

- 1: "di rodezira-ge reyoha-qi" (3 words, 5 morphemes)

- 2: "yi tohegipu-ga-ga rodezira-sa-yu" (3 words, 7 morphemes)

- 3: "tu rodezira-de-ro-pa gitopide-pe-re-qu" (3 words, 9 morphemes)

- 4: "gu rodezira-fa-ho-fu-qo deguqaso-ge-ri-re-hu" (3 words, 11 morphemes)

This whole process was repeated 10 times, to create 10 corpora, each 5,000 sentences in length, for each of the five different languages.

3.1.2 Segmentation. The same procedures were used as in Experiment 1.

\subsection{Results and discussion}

Results for Experiment 2 are shown in Fig. 3. Our first goal was to answer whether languages varying in morphological complexity, as defined by the number of morphemes per word, differ in segmentability. A regression predicting F-scores 5 from language, level, algorithm, and their interactions accounted for most variance in the

\footnotetext{
18 All F-scores are presented numerically in the online supplementary material (folder 'results', file 'res-stat-ratio-Exp.2.txt').
} 


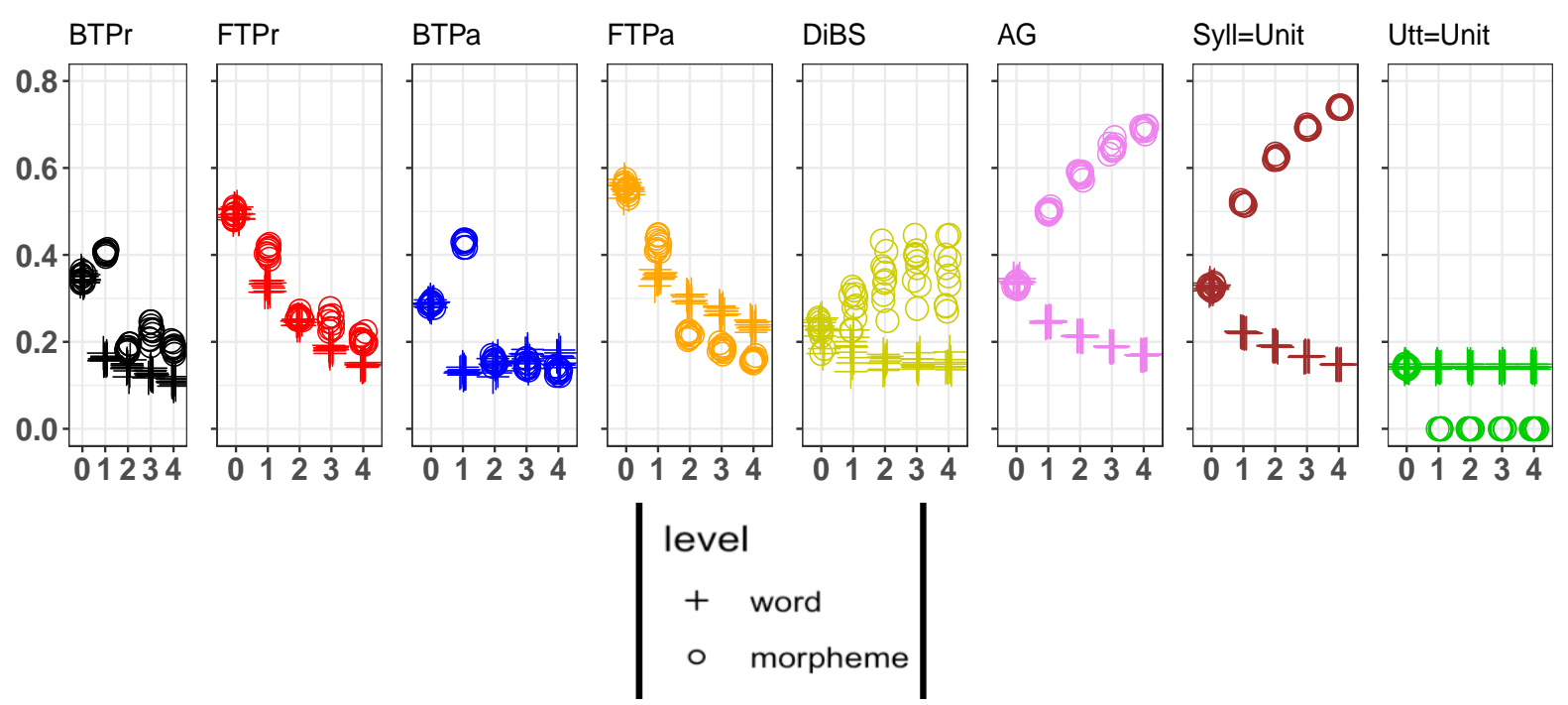

Figure 3. The y axis shows token F-scores across languages. The languages in the $\mathrm{x}$ axis are 0 (stems take no affixes), 1 (stems take one affix), 2 (stems take two affixes), 3 (stems take three affixes) and 4 (stems take four affixes). Evaluation levels are marked by shape. Color reflects algorithms (which are also used to group the data into boxes, see title of each box).

data, $R^{2}=.99(F(63,568)=720, p<.001)$ Even though the presence of significant interactions precluded a direct interpretation of the main effects, the regression confirmed a disadvantage for the most morphologically complex languages, with negative coefficients estimating the language effect ( -0.02 for language $2,-0.04$ for 3 , -0.05 for 4$)$. Thus, the answer to our first research question is that there is an effect of cross-linguistic complexity on segmentability.

In response to our second research goal, how large the language effect is compared to differences across algorithms and level, we observed that the language effect was relatively small, since the F-value for language was several times smaller than that of level and of algorithm. Two aspects of the results apparent in Fig. 3 are worth pointing out. Morpheme level scores for languages where words contain two, three, four and five

19 The function was: $\operatorname{lm}$ (token fscores $\sim$ language $*$ level $*$ algorithm $+(1 /$ subset $)$, data $).$ Token F-scores are the F-scores to be predicted by language, level, and algorithm as fixed effects, and subset as random factor. The data frame contains 640 observations (4 languages x 2 levels $\mathrm{x} 10$ subsets $\mathrm{x} 8$ algorithms). Regression results for all factors and their interactions can be found at https://osf.io/e8d2r/?view_only=f9d7b6a307734268bd8a515c55255b69, file 'supplementary_material.pdf', p. 10-12. 
morphemes, were in general higher than word level results for the same language, meaning that morphemes were heavily segmented out in morphologically complex languages. AG and DiBS morpheme-level results increased with language complexity, reaching and even surpassing the results for the language with no affixes. These two observations highlight the large effect of the evaluation level and reveal an interaction between level and language. Similar to Experiment 1, the difficulty of segmenting morphologically complex languages was mitigated when we considered morphemes instead of words as a segmentation goal.

In sum, we observed the expected differences in performance as a function of language, as well as important effects of level and algorithm. The results of Experiment 2 support our conclusions from Experiment 1: Languages varying in morphological complexity vary in segmentability. Overall, word segmentation performance for the simplest language where words and morphemes coincide was better than word performance for the other languages, which contain 1-4 affixes per stem. However, the strength of the language effect varied across algorithms, and was even reversed in some conditions, exactly as we observed in Experiment 1. AG and Syll=Unit on the morpheme level yielded considerably higher results for the complex languages than for the simpler ones. AG looks for minimal recombinable units, and it broke words up into their component morphemes. Syll=Unit adds boundaries after each syllable, and it segmented out one-syllable long morphemes. These strategies might work well for complex languages like Chintang (more details on the algorithms' performance are in the Appendix, Section 6).

The language effect was again smaller than the effects found for the other two factors, namely level and algorithm, even though the range of cross-linguistic variance in this Experiment was huge, much larger than that found in Experiment 1's natural language corpora. 


\section{Experiment 3: Artificial languages varying in the distribution of affix number}

We implemented one more set of artificial languages in order to observe the effect of morphological complexity in controlled environments. One limitation of Experiment 2 is that languages were more internally homogeneous in terms of complexity than human languages typically are. There is no human language in which all content words always have exactly the same number of affixes. We relaxed this assumption while maintaining differences in complexity in our Experiment 3.

Specifically, the languages in this experiment exhibit a distribution of complexity; they were created to differ in the distribution of affix numbers, with all artificial languages having words that contain between zero and four affixes but varying in how frequent different affix numbers were. In our baseline language, the probability distribution was flat, with $20 \%$ probability for each option (zero to four affixes). In a simpler language, more mass was allocated to a lower number of affixes. Few words in the simplest language have more than one affix, but they exist. Finally, a more complex language was created with more mass allocated to a higher number of affixes. The complex language nonetheless has some words with just one affix.

This corpus provides a deeper understanding of the effects of natural language morphology to segmentation, when compared to the "extreme" Experiment 2, where e.g. all words of the most complex language had the same large number of affixes. The same questions and predictions of Experiments 1 and 2 are revisited in this experiment: We ask whether languages varying in morphological complexity differ in segmentability, and how large this effect is when compared to differences across algorithms and evaluation level.

We predict that the cross-linguistic differences are less marked in this Experiment compared to the previous one, exactly because there are similarities in structure across these three languages. 
Table 4

\begin{tabular}{|c|r|r|r|}
\hline Mean subset stats & $\mathrm{S}$ & $\mathrm{B}$ & $\mathrm{C}$ \\
\hline Nb utterances & 5,000 & 5,000 & 5,000 \\
\hline Nb word tokens & $12470(106)$ & $12,518(69)$ & $12,532(64)$ \\
Nb word types & $5,336(47)$ & $6,481(43)$ & $7,174(25)$ \\
Nb word hapaxes & $4,467(51)$ & $5,903(65)$ & $6,922(27)$ \\
Nb phoneme tokens & $70,240(676)$ & $80,574(809)$ & $90,452(645)$ \\
Nb morpheme tokens & $22,576(141)$ & $27,598(233)$ & $32,452(195)$ \\
\hline
\end{tabular}

Means (and standard deviation) of corpus features across the ten subsets of artificial languages $S$ (simpler), B (base) and C (more complex). "Nb" stands for number.

\subsection{Methods}

4.1.1 Languages. As in Experiment 2, we created languages with a lexicon of 1,000 items, of which 1\% were one-syllable long function words, and the remaining were stems one- to four-syllables in length, randomly split into two types that selected different affix paradigms. The syllable inventory, distribution of sentence length (1-4 words), length of corpora (5,000 sentences), were also kept constant, and 10 subsets were generated for each language.

Unlike in Experiment 2, however, all languages had some affixes, and stems could take between 0 and 4 affixes. The three languages we created varied in terms of the distribution of the number of affixes accompanying a stem. In the base language (B), it was equally likely for stems to have 0 to 4 affixes (i.e., $20 \%$ of chances for each). In the simpler language (S), the distribution was tilted towards fewer affixes: $35 \%$ likelihood of having 0 affixes, $25 \%$ of having 1, $20 \%$ of having $2,10 \%$ of having 3 , and $10 \%$ of having 4 affixes. The more complex language (C) had the opposite trend: $10 \%$ likelihood of having 0 affixes, $10 \%$ of having 1, $20 \%$ of having $2,25 \%$ of having 3 , and $35 \%$ of having 4 affixes. Table 4 shows some basic statistics of the languages.

4.1.2 Segmentation. The same procedures were used as in Experiment 1. 


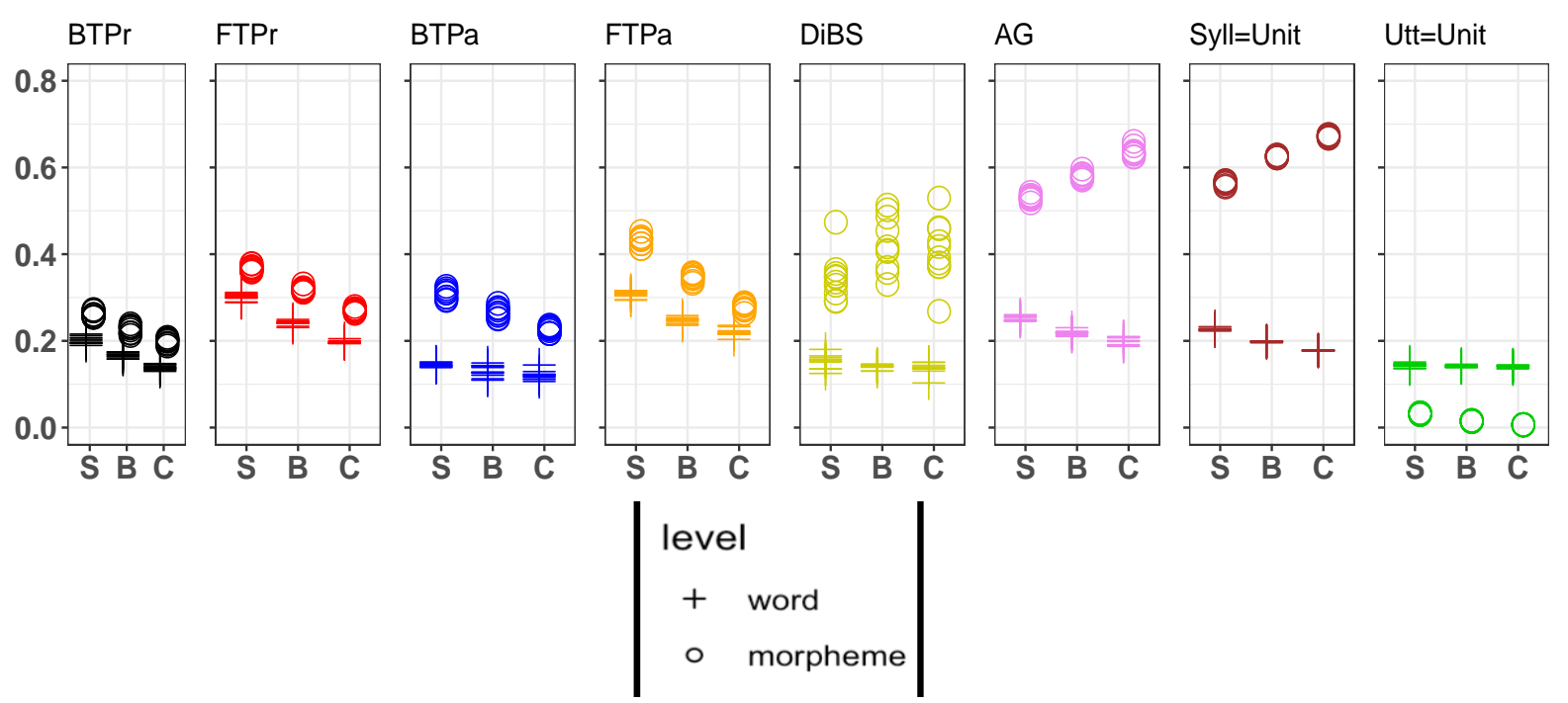

Figure 4. The y axis contains token F-scores across languages. The languages in the $\mathrm{x}$ axis are S(imple), B(ase) and C(omplex). Evaluation levels are marked by shape. Color reflects algorithms (which are also used to group the data into boxes, see title of each box).

\subsection{Results and discussion}

Results for Experiment 3 are shown in Fig. 4. In general, more similarities in segmentability across languages were found here than in Experiment 2, as predicted, since languages were more similar to each other here than in Experiment 2.

Bearing on our first research question, a regression predicting F-scores ${ }^{20}$ from language (S, B and C), level, algorithm, and their interactions accounted for most variance in the data, $R^{2}=.99(F(47,945)=432, p<.001){ }^{21}$ The regression yielded a small disadvantage for the more morphologically complex languages overall, with negative coefficients estimating the language effect (-0.03 for B and -0.06 for $\mathrm{C}$ ). This is because all models, when evaluated on words, performed better for the simplest

\footnotetext{
20 All F-scores are presented numerically in the online supplementary material (folder 'results', file 'res-stat-prob-Exp.3.txt').

21 The function was: $\operatorname{lm}($ token fscores $\sim$ language $*$ level $*$ algorithm $+(1 /$ subset $)$, data $)$. Token F-scores are the F-scores to be predicted by language, level, and algorithm as fixed effects, and subset as random factor. The data frame contains 480 observations (3 languages x 2 levels x 10 subsets x 8 algorithms). Regression results for all factors and their interactions can be found at https://osf.io/e8d2r/?view_only=f9d7b6a307734268bd8a515c55255b69, file
} 'supplementary_material.pdf', p. 12-14. 
languages. However the significant interactions with algorithm and level precluded a direct interpretation of this main effect, as detailed in the next paragraph.

Regarding our second research goal, we observed that language effects were relatively small, since the F-value for language was half the size of level and algorithm. Two more aspects of the results are worth pointing out. First, similarly to Experiment 2, scores for all three languages improved when evaluating on morphemes. Second, for most models, morphologically complex languages were easier to segment on the morpheme level. These aspects confirm that the strength of the language effect largely depends on its interactions with the other two important factors, namely evaluation level and algorithm type.

\section{Overall discussion}

These three studies are the first to openly state that there could be a difference in segmentability due to morphological complexity, and to carefully study it. They are also the first to systematically look at performance differences across level and quantitatively document them. Our work follows an innovative scientific trend to go beyond merely showing an effect, but instead attempt to accurately measure effect sizes, and to put factors into context in order to assess their importance. We can now highlight that this segmentability difference as a function of morphological complexity is actually quite small (compared to differences when evaluating on morphemes or words, and differences across algorithms), and this is observed after comparing several different segmentation strategies on the same languages. Differences within-language across algorithm and level are larger than differences across languages.

These conclusions are informed from segmenting both natural (Experiment 1) and artificial languages (Experiments 2 and 3). When comparing two natural languages, we could not be certain that all segmentability differences were due to the specific factor we had identified, and we were further uncertain whether its relatively small size may not be due to constrained variation found in the comparison of only two languages. Once everything else was controlled for via artificial languages, the effect of 
morphological complexity was clear and could not be attributed to any confounds. However, particularly in the context of Experiment 2, where languages differ maximally in morphological complexity - more than what any human natural languages could differ-, the effect of morphological complexity remained small, and certainly smaller than that of level and algorithm.

The artificial language results were also informative on the algorithm performance; the performance range of the algorithms was similar across all three experiments, highlighting the relevance of our artificial language results for broader generalization to natural languages. Additionally, this suggested that differences across algorithms are massive - even when corpora are perfectly controlled. These findings invite experimenters on language acquisition to focus more on the algorithm effects, rather than language effects in future studies, since language effects are bound to be small.

Our three experiments also revealed that there are crucial interactions: all algorithms used here (even sublexical ones) interacted with language and level, and there was no one-size-fits-all strategy. One algorithm may perform better for a given language and level (e.g. segmentation of words by a lexical algorithm might not work well for a complex language), whereas another algorithm may be optimal for a different language and level combination.

These interactions allow us to make an important set of predictions for infant language acquisition. One possibility is that infants are endowed with a single algorithm for segmentation. If that is the case, then we should find that infants learning certain languages (notably those with complex inflectional systems) should be delayed in their word segmentation compared to infants learning simpler languages. This prediction cannot be tested by creating artificial languages, because it is a prediction about how infants extract words and morphemes from their native language. Instead, it should be tested by assessing infants' recognition of words and morphemes in their language, particularly those that do not frequently occur in isolation (for example on Hungarian, Ladányi, Kovács and Gervain, 2020). WordBank (Frank, Braginsky, Yurovsky \& Marchman, 2017) is an open repository for developmental vocabulary data that could 
be used for these analyses. Should such an experiment not show systematic differences across language backgrounds, then a second hypothesis needs to be envisaged.

According to this second hypothesis, infants are not endowed with a single algorithm, but have in their toolkit algorithms covering the same range of approaches studied here, or an even greater one. In this case, however, the learning problem becomes even more complex, because infants need to have a "meta-algorithm", which allows them to choose among alternative algorithms. There has been a proposal of how this could be done: Fourtassi and Dupoux (2014) argue that infants could assess an algorithms' performance by splitting their input into subsets, and learning words (or, presumably, morphemes) from each half separately: Better algorithms should lead to a more consistent lexicon. If this proposal is on the right track, then we should observe that younger infants have access to a wider array of segmentation algorithms than older infants, a prediction that should be tested using artificial languages learned in the laboratory. What one expects to observe, then, is that younger infants can break up an artificial stream using a wide array of strategies (including lexical and sublexical ones); whereas older infants fail when presented with artificial languages that can only be segmented when using one specific strategy, which is not used in the infant's native language.

While doing this work, we realized that the experimental literature in infancy has not yet attempted to provide proof-of-principle evidence for the presence of all these segmentation algorithms. In particular, we think it would be important to check whether infants optimize the lexicon in the input they hear, as predicted if they have a strategy like Adaptor Grammar. Another promising direction would involve checking whether infants may at some stage treat each syllable as a separate segmentation unit, as in one of our baselines (Syll=Unit). In fact, other work shows that segmenting at every syllable boundary leads to the highest F-scores in English, even outperforming Adaptor Grammar (but not in Spanish or Catalan, where Adaptor Grammar outperforms the syllable-based segmentation in terms of Model Word Accuracy; Anonymized, 2020). Incidentally, these two strategies seem to perform quite well, and 
may thus be viable strategies for natural language acquisition (Loukatou, Moran, Blasi, Stoll \& Cristia, 2019).

Before closing, we would like to bring up a number of limitations of this paper. First, working from text-like phonologization simplifies the learning problem to a certain extent, assuming that infants represent perfectly the sequences of phonemes in the speech they hear. Even though there is some literature looking at word segmentation from speech (Ludusan et al., 2015), this task remains challenging for computational modeling.

Additionally, we would like to mention that the algorithms were evaluated on words, as defined by their conventional orthographic representations. However, wordhood and morphemehood are debated issues in linguistics and psycholinguistics, without cross-linguistically valid definitions (see also Hay and Baayen, 2005 for differences in salience across morphemes).

We also conducted two artificial language experiments, where we created languages differing in morphological complexity while keeping their lexicon size constant. We acknowledge that this is a simplification; the lexicon size of natural languages varies, and it may even interact with their level of morphology.

Finally, we studied languages that differ in morphological synthesis, admittedly considering only one dimension of morphological complexity. We suggest that the level of allomorphy, meaning how many different realizations exist for a single morpheme, or the fusion of the affixes with each other, could also affect segmentation. Further research is needed to show the effects of these morphological aspects, although this would ideally involve recovery of morphological paradigms, and not just segmentation as done here.

In sum, our work constitutes an important step towards documenting segmentation differences and similarities across languages, and considering possible evaluation targets for language acquisition segmentation models. We hope future research retains our strategy of employing a range of plausible algorithms and evaluating on different linguistic levels. 
References

Aikhenvald, A. (2007). Typological distinctions in word-formation. In Language Typology and Syntactic Description, Volume III: Grammatical Categories and the Lexicon (pp. 1-64). Cambridge: Cambridge University Press.

Allen, S. (1996). Aspects of argument structure acquisition in inuktitut. John Benjamins Publishing.

Anonymized. (2020). Is there a bilingual disadvantage for word segmentation? a computational modeling approach.

Bane, M. (2008). Quantifying and measuring morphological complexity. In Proceedings of the 26th west coast conference on formal linguistics (pp. 69-76). Cascadilla Proceedings Project Somerville, MA.

Bartlett, S., Kondrak, G. \& Cherry, C. (2009). On the syllabification of phonemes. In Proceedings of Human Language Technologies: The 2009 annual conference of the North American chapter of the Association for Computational Linguistics (pp. 308-316). Association for Computational Linguistics.

Batchelder, E. O. (2002). Bootstrapping the lexicon: A computational model of infant speech segmentation. Cognition, 83(2), 167-206.

Bates, E., Marchman, V., Thal, D., Fenson, L., Dale, P., Reznick, J. S., ... Hartung, J. (1994). Developmental and stylistic variation in the composition of early vocabulary. Journal of Child Language, 21(1), 85-123.

Bentz, C., Soldatova, T., Koplenig, A. \& Samardžić, T. (2016). A comparison between morphological complexity measures: Typological data vs. language corpora. Proceedings of the Workshop on Computational Linguistics for Linguistic Complexity (CL4LC). Osaka, Japan, part of CoLing 2016, 142-153.

Bernard, M., Thiolliere, R., Saksida, A., Loukatou, G., Larsen, E., Johnson, M., ... Cristia, A. (2018). Wordseg: Standardizing unsupervised word form segmentation from text. Behavior research Methods.

Bickel, B., Banjade, G., Gaenszle, M., Lieven, E., Paudyal, N. P., Rai, I. P., .. Stoll, S. (2007). Free prefix ordering in Chintang. Language, 83(1), 43-73. 
Bickel, B. \& Nichols, J. (2007). Inflectional morphology. In T. Shopen (Ed.), Language typology and syntactic description (Vol. 3, pp. 169-240). 2nd edition. Cambridge: Cambridge University Press.

Bickel, B. \& Nichols, J. (2013a). Fusion of selected inflectional formatives. In M. S. Dryer \& M. Haspelmath (Eds.), The World Atlas of Language Structures Online. Leipzig: Max Planck Institute for Evolutionary Anthropology. Retrieved from http://wals.info/chapter/20

Bickel, B. \& Nichols, J. (2013b). Inflectional synthesis of the verb. In M. S. Dryer \& M. Haspelmath (Eds.), The World Atlas of Language Structures Online. Leipzig: Max Planck Institute for Evolutionary Anthropology. Retrieved from http://wals.info/chapter/22

Bickel, B., Nichols, J., Zakharko, T., Witzlack-Makarevich, A., Hildebrandt, K., Rießler, M., ... John, B. L. (2017). The AUTOTYP typological databases. Version 0.1. 0. Online: https://github. com/autotyp/autotyp-data/tree/0.1. 0. Bickel, B. \& Zúñiga, F. (2017). The 'word' in polysynthetic languages: Phonological and syntactic challenges. In M. Fortescue, M. Mithun \& N. Evans (Eds.), The Oxford Handbook of Polysynthesis (pp. 158-185). Oxford: Oxford University Press.

Black, A. \& Bergmann, C. (2017). Quantifying infants' statistical word segmentation: A meta-analysis. In Proceedings of the 39th annual conference of the Cognitive Science Society (pp. 124-129).

Blanchard, D., Heinz, J. \& Golinkoff, R. (2010). Modeling the contribution of phonotactic cues to the problem of word segmentation. Journal of Child Language, 37(3), 487-511.

Bortfeld, H., Morgan, J. L., Golinkoff, R. M. \& Rathbun, K. (2005). Mommy and me: Familiar names help launch babies into speech-stream segmentation. Psychological Science, 16(4), 298-304.

Boruta, L., Peperkamp, S., Crabbé, B. \& Dupoux, E. (2011). Testing the robustness of online word segmentation: Effects of linguistic diversity and phonetic variation. In 
Proceedings of the 2nd workshop on Cognitive Modeling and Computational Linguistics (pp. 1-9). Association for Computational Linguistics.

Brent, M. R. (1997). Toward a unified model of lexical acquisition and lexical access. Journal of psycholinguistic research, 26(3), 363-375.

Brent, M. R. \& Cartwright, T. A. (1996). Distributional regularity and phonotactic constraints are useful for segmentation. Cognition, 61(1-2), 93-125.

Brent, M. R. \& Siskind, J. M. (2001). The role of exposure to isolated words in early vocabulary development. Cognition, 81(2), B33-B44.

Caines, A., Altmann-Richer, E. \& Buttery, P. (2019). The cross-linguistic performance of word segmentation models over time. Journal of Child Language, 46(6), $1169-1201$.

Coe, R. (2002). It's the effect size, stupid: What effect size is and why it is important.

Çöltekin, Ç. (2011). Catching words in a stream of speech: Computational simulations of segmenting transcribed child-directed speech (Doctoral dissertation, University of Groningen).

Daland, R. (2009). Word segmentation, word recognition, and word learning: A computational model of first language acquisition (Doctoral dissertation, Northwestern University).

Daland, R. \& Pierrehumbert, J. B. (2011). Learning diphone-based segmentation. Cognitive science, 35(1), 119-155.

Davis, M. H., Marslen-Wilson, W. D. \& Gaskell, M. G. (2002). Leading up the lexical garden path: Segmentation and ambiguity in spoken word recognition. Journal of Experimental Psychology: Human Perception and Performance, $28(1), 218$.

DeKeyser, R. M. (2005). What makes learning second-language grammar difficult? A review of issues. Language Learning, 55(S1), 1-25.

Dixon, R. M. \& Aikhenvald, A. Y. (2002). Word: A cross-linguistic typology. Cambridge: Cambridge University Press.

Dryer, M. S. \& Haspelmath, M. (Eds.). (2013). WALS Online. Leipzig: Max Planck Institute for Evolutionary Anthropology. Retrieved from http://wals.info/ 
Endress, A. D. \& Hauser, M. D. (2010). Word segmentation with universal prosodic cues. Cognitive Psychology, 61, 177-199.

Fleck, M. M. (2008). Lexicalized phonotactic word segmentation. In Proceedings of the joint meeting of the Association for Computational Linguistics and the Human Language Technology Conference (pp. 130-138).

Fourtassi, A., Börschinger, B., Johnson, M. \& Dupoux, E. (2013). WhyisEnglishsoeasytosegment. In Proceedings of the fourth annual workshop on Cognitive Modeling and Computational Linguistics (pp. 1-10).

Fourtassi, A. \& Dupoux, E. (2014). A rudimentary lexicon and semantics help bootstrap phoneme acquisition. In Proceedings of the eighteenth conference on computational natural language learning (pp. 191-200).

Frank, M. C., Braginsky, M., Yurovsky, D. \& Marchman, V. A. (2017). Wordbank: An open repository for developmental vocabulary data. Journal of child language, $44(3), 677$.

Gaenszle, M., Bickel, B., Banjade, G., Lieven, E., Paudyal, N., Rai, A., ... Stoll, S. (2005). Research report: the Chintang and Puma Documentation Project (CPDP). European Bulletin of Himalayan Research, (28), 95-103.

Gambell, T. \& Yang, C. (2005). Word segmentation: Quick but not dirty. Unpublished manuscript.

Gervain, J. \& Erra, R. G. (2012). The statistical signature of morphosyntax: A study of Hungarian and Italian infant-directed speech. Cognition, 125(2), 263-287.

Goldwater, S., Griffiths, T. L. \& Johnson, M. (2009). A bayesian framework for word segmentation: Exploring the effects of context. Cognition, 112(1), 21-54.

Gow, D. \& Gordon, P. (1995). Lexical and prelexical influences on word segmentation: Evidence from priming. Journal of Experimental Psychology: Human perception and performance, $21(2), 344$.

Hallé, P. A., Durand, C. \& de Boysson-Bardies, B. (2008). Do 11-month-old French infants process articles? Language and Speech, 51(1-2), 23-44. 
Haspelmath, M. (2011). The indeterminacy of word segmentation and the nature of morphology and syntax. Folia Linguistica, 45 (1), 31-80.

Hay, J. B. \& Baayen, R. H. (2005). Shifting paradigms: Gradient structure in morphology. Trends in cognitive sciences, 9(7), 342-348.

Hinds, J. (1986). Japanese. London: Routledge.

Höhle, B. \& Weissenborn, J. (2003). German-learning infants' ability to detect unstressed closed-class elements in continuous speech. Developmental Science, $6(2), 122-127$.

Johnson, E. K. \& Jusczyk, P. W. (2001). Word segmentation by 8-month-olds: When speech cues count more than statistics. Journal of memory and language, $44(4)$, $548-567$.

Johnson, M. (2008). Unsupervised word segmentation for Sesotho using Adaptor Grammars. In Proceedings of the tenth meeting of Association for Computational Linguistics Special Interest Group on Computational Morphology and Phonology (pp. 20-27). Association for Computational Linguistics.

Johnson, M. \& Demuth, K. (2010). Unsupervised phonemic Chinese word segmentation using Adaptor Grammars. In Proceedings of the 23rd International Conference on Computational Linguistics (pp. 528-536). Association for Computational Linguistics.

Johnson, M., Griffiths, T. L. \& Goldwater, S. (2007). Adaptor Grammars: A framework for specifying compositional nonparametric Bayesian models. In Advances in neural information processing systems (pp. 641-648).

Jusczyk, P. W., Houston, D. M. \& Newsome, M. (1999). The beginnings of word segmentation in english-learning infants. Cognitive psychology, 39(3-4), 159-207.

Jusczyk, P. W., Jusczyk, A. M., Kennedy, L. J., Schomberg, T. \& Koenig, N. (1995). Young infants' retention of information about bisyllabic utterances. Journal of Experimental Psychology: Human Perception and Performance, 21 (4), 822-836.

Kahn, D. (2015). Syllable-based generalizations in english phonology. Routledge. 
Kastner, I. \& Adriaans, F. (2017). Linguistic constraints on statistical word segmentation: The role of consonants in Arabic and English. Cognitive Science, 42(2), 494-518.

Keren-Portnoy, T., Vihman, M. \& Fisher, R. L. (2019). Do infants learn from isolated words? an ecological study. Language Learning and Development, 15(1), 47-63.

Ketrez, F. N. \& Aksu-Koç, A. (2009). Early nominal morphology in Turkish: Emergence of case and number. In M. Voeikova \& U. Stephany (Eds.), The development of nominal inflection in first language acquisition: A cross-linguistic perspective (pp. 15-48). Berlin: Mouton de Gruyter.

Kuno, S. (1973). The structure of the Japanese language. Cambridge, MA: MIT press.

Ladányi, E., Kovács, Á. M. \& Gervain, J. (2020). How 15-month-old infants process morphologically complex forms in an agglutinative language? Infancy, 25(2), $190-204$.

Lignos, C. (2012). Infant word segmentation: An incremental, integrated model. In Proceedings of the West Coast Conference on Formal Linguistics (237-247)).

Loukatou, G., Le Normand, M. \& Cristia, A. (2019). Is it easier to segment words from infant- than adult-directed speech? Modeling evidence from an ecological French corpus. In Proceedings of the 41st Annual Conference of the Cognitive Science Society.

Loukatou, G., Moran, S., Blasi, D., Stoll, S. \& Cristia, A. (2019). Is word segmentation child's play in all languages? In Proceedings of the 57th annual meeting of the association for computational linguistics (pp. 3931-3937).

Loukatou, G., Stoll, S. \& Cristia, A. (2018). Computational word segmentation and code-switching: The chintang case [poster presentation]. Learning Language in Humans and in Machines (L2HM). doi:10.17605/OSF.IO/UVEBD

Ludusan, B., Seidl, A., Dupoux, E. \& Cristia, A. (2015). Motif discovery in infant-and adult-directed speech. In Proceedings of the sixth workshop on Cognitive Aspects of Computational Language Learning (pp. 93-102). 
Marquis, A. \& Shi, R. (2015). The beginning of morphological learning: Evidence from verb morpheme processing in preverbal infants. Cognitive Science Perspectives on Verb Representation and Processing, 281-297.

Mattys, S. L. \& Jusczyk, P. W. (2001). Phonotactic cues for segmentation of fluent speech by infants. Cognition, 78(2), 91-121.

Mattys, S. L., Jusczyk, P. W., Luce, P. A. \& Morgan, J. L. (1999). Phonotactic and prosodic effects on word segmentation in infants. Cognitive psychology, 38(4), 465-494.

McWhorter, J. H. (2001). The worlds simplest grammars are creole grammars. Linguistic typology, 5(2-3), 125-166.

Mersad, K. \& Nazzi, T. (2012). When mommy comes to the rescue of statistics: Infants combine top-down and bottom-up cues to segment speech. Language Learning and Development, 8(3), 303-315.

Miestamo, M. et al. (2008). Grammatical complexity in a cross-linguistic perspective. Language complexity: Typology, contact, change, 23, 41.

Mintz, T. H. (2013). The segmentation of sub-lexical morphemes in English-learning 15-month-olds. Frontiers in Psychology, 4(24).

Miyata, S. (2004a). Japanese: Aki Corpus. Pittsburgh PA: TalkBank, 1-59642-055-3.

Miyata, S. (2004b). Japanese: Ryo corpus. Pittsburgh PA: TalkBank, 1-59642-056-1.

Miyata, S. (2004c). Japanese: Tai corpus. Pittsburgh, PA: TalkBank, 1-59642-057-X.

Miyata, S. \& Naka, N. (1998). Wakachigaki Guideline for Japanese: WAKACHI98 v.1.1. In The Japanese Society for Educational Psychology Forum Report No. FR-98-003, The Japanese Association of Educational Psychology.

Miyata, S. \& Naka, N. (2006). JMOR03. Retrieved from http://childes.psy.cmu.edu/morgrams/jpn.zip.

Miyata, S. \& Naka, N. (2014). JMOR06.2: The Japanese morphological analysis program based on CLAN.

Miyata, S. \& Nisisawa, H. (2009). Japanese-MiiPro-Asato Corpus. Pittsburgh, PA: TalkBank, 1-59642-474-5. 
Miyata, S. \& Nisisawa, H. (2010). Japanese-MiiPro-Tomito Corpus. Pittsburgh, PA: TalkBank, 1-59642-474-5.

Monaghan, P. \& Christiansen, M. H. (2010). Words in puddles of sound: Modelling psycholinguistic effects in speech segmentation. Journal of child language, 37(3), $545-564$.

Moran, S. \& Cysouw, M. (2018). The unicode cookbook for linguists: Managing writing systems using orthography profiles.

Moran, S., Schikowski, R., Pajović, D., Hysi, C. \& Stoll, S. (2016). The ACQDIV database: Min (d) ing the ambient language. In Proceedings of the tenth international conference on Language Resources and Evaluation (lrec 2016) (pp. 4423-4429).

Ngon, C., Martin, A., Dupoux, E., Cabrol, D., Dutat, M. \& Peperkamp, S. (2013). (Non) words, (non) words, (non) words: Evidence for a protolexicon during the first year of life. Developmental Science, 16(1), 24-34.

Nichols, J., Witzlack-Makarevich, A. \& Bickel, B. (2013). The AUTOTYP genealogy and geography database: 2013 release. Zurich: University of Zurich.

Nisisawa, H. \& Miyata, S. (2009). Japanese-MiiPro-Nanami Corpus. Pittsburgh, PA: TalkBank, 1-59642-475-3.

Nisisawa, H. \& Miyata, S. (2010). Japanese-MiiPro-ArikaM Corpus. Pittsburgh, PA: TalkBank, 1-59642-475-3.

Norris, D., McQueen, J. M., Cutler, A. \& Butterfield, S. (1997). The possible-word constraint in the segmentation of continuous speech. Cognitive Psychology, 34(3), $191-243$.

Pearl, L. \& Phillips, L. (2018). Evaluating language acquisition models: A utility-based look at Bayesian segmentation. In A. Villavicencio \& T. Poibeau (Eds.), Language, cognition, and computational models (pp. 185-224). Cambridge: Cambridge University Press. 
Perfors, A. \& Navarro, D. J. (2012). What Bayesian modelling can tell us about statistical learning: What it requires and why it works. Statistical Learning and Language Acquisition, 1, 383-408.

Phillips, L. \& Pearl, L. (2014a). Bayesian inference as a cross-linguistic word segmentation strategy: Always learning useful things. In Proc. of 5th workshop on Cognitive Aspects of Computational Language Learning (pp. 9-13).

Phillips, L. \& Pearl, L. (2014b). Bayesian inference as a viable cross-linguistic word segmentation strategy: It's all about what's useful. In Proceedings of the Cognitive Science Society (pp. 2775-2780).

Phillips, L. \& Pearl, L. (2015). The utility of cognitive plausibility in language acquisition modeling: Evidence from word segmentation. Cognitive Science, 39(8), $1824-1854$.

Phillips, L. \& Pearl, L. (2018). Syllabic bayesian word segmenter. https://github.com/lawphill/phillips-pearl2014.

R Core Team. (2013). R: A language and environment for statistical computing. $\mathrm{R}$ Foundation for Statistical Computing. Vienna, Austria. Retrieved from http://www.R-project.org/

Roy, D. K. \& Pentland, A. P. (2002). Learning words from sights and sounds: A computational model. Cognitive Science, 26(1), 113-146.

Ruokolainen, T., Kohonen, O., Sirts, K., Grönroos, S.-A., Kurimo, M. \& Virpioja, S. (2016). A comparative study of minimally supervised morphological segmentation. Computational Linguistics, 42(1), 91-120.

Ruzsics, T. \& Samardzic, T. (2017). Neural sequence-to-sequence learning of internal word structure. In Proceedings of the 21st Conference on Computational Natural Language Learning (CoNLL 2017) (pp. 184-194).

Saffran, J. R., Aslin, R. N. \& Newport, E. L. (1996). Statistical learning by 8-month-old infants. Science, 1926-1928.

Saffran, J. R., Newport, E. L. \& Aslin, R. N. (1996). Word segmentation: The role of distributional cues. Journal of Memory and Language, 35 (4), 606-621. 
Saksida, A., Langus, A. \& Nespor, M. (2017). Co-occurrence statistics as a language-dependent cue for speech segmentation. Developmental Science, 20(3), $1-11$.

Samardzic, T., Schikowski, R. \& Stoll, S. (2015). Automatic interlinear glossing as two-level sequence classification. In Proceedings of the 9th SIGHUM Workshop on Language Technology for Cultural Heritage, Social Sciences, and Humanities (LaTeCH) (pp. 68-72).

Schiering, R., Bickel, B. \& Hildebrandt, K. A. (2010). The prosodic word is not universal, but emergent. Journal of Linguistics, 46 (3), 657-709.

Schikowski, R., Moran, S. \& Stoll, S. (2018). Manual for the ACQDIV corpus. https://github.com/acqdiv/corpus_manual/blob/master/corpus_manual.pdf

Seidl, A., Cristià, A., Bernard, A. \& Onishi, K. H. (2009). Allophonic and phonemic contrasts in infants' learning of sound patterns. Language Learning and Development, 5(3), 191-202.

Shi, R. \& Gauthier, B. (2005). Recognition of function words in 8-month-old French-learning infants. The Journal of the Acoustical Society of America, 117(4), $2426-2427$.

Shi, R. \& Lepage, M. (2008). The effect of functional morphemes on word segmentation in preverbal infants. Developmental Science, 11(3), 407-413.

Shi, R., Marquis, A. \& Gauthier, B. (2006). Segmentation and representation of function words in preverbal French-learning infants. In Proceedings of the 30th annual boston university conference on language development (Vol. 2, pp. 549-560). Cascadilla Somerville, MA.

Shibatani, M. (1990). Japanese. In B. Comrie (Ed.), The world's major languages (pp. 855-880). London Sydney: Croom Helm.

Shosted, R. K. (2006). Correlating complexity: A typological approach. Linguistic Typology, 10(1), 1-40. 
Shukla, M., White, K. S. \& Aslin, R. N. (2011). Prosody guides the rapid mapping of auditory word forms onto visual objects in 6-mo-old infants. Proceedings of the National Academy of Sciences, 108(15), 6038-6043.

Skoruppa, K., Pons, F., Christophe, A., Bosch, L., Dupoux, E., Sebastián-Gallés, N., ... Peperkamp, S. (2009). Language-specific stress perception by 9-month-old french and spanish infants. Developmental Science, 12(6), 914-919.

Slobin, D. I. (1985). The crosslinguistic study of language acquisition: Theoretical issues. Hillsdale, NJ: Lawrence Erlbaum Associates.

Slobin, D. I. (2014). Before the beginning: The development of tools of the trade. Journal of Child Language, 41(S1), 1-17.

Stoll, S. (2015). Crosslinguistic approaches to language acquisition. In E. L. Bavin \& L. R. Naigles (Eds.), The Cambridge Handbook of Child Language (pp. 107-134). Cambridge Handbooks in Language and Linguistics. doi:10.1017/CBO9781316095829.006

Stoll, S. \& Bickel, B. (2013). Capturing diversity in language acquisition research. In B. Bickel, L. Grenoble, D. Peterson \& A. Timberlake (Eds.), Language Typology and Historical Contingency: In honor of Johanna Nichols (pp. 1-22). Amsterdam: John Benjamins.

Stoll, S. \& Lieven, E. (2014). Studying language acquisition cross-linguistically. In H. Winskel \& P. Pradakannaya (Eds.), South and Southeast Asian Psycholinguistics (pp. 19-35). Cambridge, UK: Cambridge University Press.

Stoll, S., Mazara, J. \& Bickel, B. (2017). The acquisition of polysynthetic verb forms in Chintang. Handbook of Polysynthesis. Oxford: Oxford University Press.

Stoll, S. \& Schikowski, R. (2020). Child corpora. In P. Magali \& S. T. Gries (Eds.), Practical handbook of corpus linguistics. Springer.

Stoll, S., Zakharko, T., Moran, S., Schikowski, R. \& Bickel, B. (2015). Syntactic mixing across generations in an environment of community-wide bilingualism. Frontiers in Psychology, 6, 82. 
Tsujimura, N. (1996). An introduction to Japanese linguistics. Cambridge, MA: Blackwell.

Van Kampen, A., Parmaksiz, G., van de Vijver, R., Höhle, B., Gavarró, A. \& Freitas, M. (2008). Metrical and statistical cues for word segmentation: The use of vowel harmony and word stress as cues to word boundaries by 6 -and 9-month-old turkish learners. Language acquisition and development: Proceedings of GALA 2007, 2007, 313-324.

Venkataraman, A. (2001). A statistical model for word discovery in transcribed speech. Computational Linguistics, 27(3), 351-372.

Zipf, G. K. (1935). The psycho-biology of language: An introduction to dynamic philology. Oxford, England: Houghton Mifflin. 


\section{Appendix}

\subsection{Predictions across algorithms}

Performance comparison across models is not part of the key questions of this paper, but it may be of interest to some readers. We thus provide here a brief literature review for each algorithm type, and proceed with specific predictions.

With respect to lexical algorithms, most previous work finds cross-linguistic differences in word segmentation performance that could be explained on the basis of complexity differences. Batchelder (2002) compared the accuracy of a lexical segmentation algorithm (BootLex) on English, Japanese and Spanish corpora, and found that the algorithm performed best on English. Most other lexical work has employed versions of Adaptor Grammars (AG) (Goldwater et al., 2009; M. Johnson, 2008). AG finds patterns of frequent phoneme sequences in the input corpus, creates a lexicon based on these patterns at specified levels, and then uses the lexicon to segment the input. Using versions of this system, Boruta et al. (2011) documented better results for English than French, and better results for French than for Japanese. These roughly correspond to the languages' order of morphological complexity. M. Johnson (2008) found better results for English than Sesotho, which is morphologically much more complex than English.

Similarly, Fourtassi et al. (2013) segmented an English and a Japanese corpus using a probabilistic lexicon-building algorithm. Qualitative inspection showed that the algorithm broke off morphological affixes, with more oversegmentation cases for Japanese than English. These oversegmentation errors suggest that it might have segmented out morphemes, or at least functional elements, in addition to or instead of some notion of words (see also M. Johnson, 2008).

Corpora of morphologically rich languages such as Chintang contain fewer repetitions of each word type, as well as a higher proportion of forms that occur rarely or once, compared to languages with little morphology (Stoll et al., 2017) (see also Boruta et al., 2011). Lexical algorithms tend to recycle existing units, favoring repetition. They might detect frequently occurring word parts such as morphemes, 
instead of words e.g. they could detect a root separately from its suffixes. AG, in particular, finds the most likely segmentation using a lexicon, whose types have been assigned probabilities based on their frequency distributions. Thus, it might break words up into their component morphemes. This behavior would be rewarded when evaluated on morpheme boundaries, but it would be penalized when evaluated on word boundaries, and the model would heavily oversegment. Since AG needs access to the lexicon, it might thus be affected by cross-linguistic differences. It may further segment out morphemes in complex languages, as they are the basic building blocks of the language, instead of entire words, whose form varies.

Previous work using sublexical algorithms also revealed differences in cross-linguistic performance, which can be attributed to complexity differences in some cases. Saksida et al. (2017) used a set of TP algorithms on cross-linguistic corpora, and found higher scores for English and Dutch than for Japanese, Polish and Hungarian. Gervain and Erra (2012) documented better results for the less complex Italian than the more complex Hungarian, although this language performance was reversed when backward transitional probabilities were used.

Based on qualitative inspection, Gervain and Erra $(2012)$ commented that there may be more oversegmentation in Hungarian. TP uses local statistics and does not have access to lexical and morphological composition. However, local statistics could be informative on morpheme boundaries, rendering TP sensitive to complexity differences up to a point; since most morphemes are combined with different stems, their transitional probabilities are low and they may be segmented. In sum, our re-interpretation of previous work is generally consistent with complexity effects when evaluating at the word level for both AG and TP.

There is not a lot of previous cross-linguistic work on DiBS that can inform language morphological complexity differences. Since DiBS is an algorithm based on phonotactics, there might not be large cross-linguistic differences for the languages in our study, which vary mainly in morphology. Segmentation baselines have also rarely been used in multiple languages, so we cannot derive predictions based on previous 
work.

Predictions when evaluating at the morpheme level are not clear for all algorithms, since we cannot use previous literature to make them, but we suspect that morpheme evaluation could reduce complexity differences across languages.

\subsection{Interpretation of results across algorithms}

We now turn to the results. Focusing first on AG, its performance was similar to our predictions. It showed distinctive oversegmentation patterns compared to DiBS and TP, particularly when evaluating on words. AG might thus work well for languages like Chintang, improving performance on the morpheme level. Indeed, AG-morpheme achieved higher scores than DiBS and TP for the most complex languages. As for comparisons with previous work, we found that our AG-word scores were much lower than the .77 documented for English (Fourtassi et al., 2013).

Turning to TP, we did not observe much difference between forward and backward alternatives (cf. Gervain and Erra, 2012 for arguments that this parameter should matter for languages varying in head direction, and Saksida et al., 2017 for other data showing that it may not), or between absolute thresholds and relative thresholds with locally minimal values. However, it should be noted that, specifically in Experiment 1 with natural languages, TPr-morpheme scores were lower than TPr-word scores; this is reasonable given that in the TPr implementation a boundary can only be posited in relatively long strings of syllables (see also Gambell and Yang, 2005). With respect to comparisons with previous work, the best performance for TP in this paper was .63, well below the .85 recorded for English by Saksida et al. (2017).

TP implementations were sensitive to morpheme boundaries. This was especially true for our artificial languages, where each affix has the length of a syllable. DiBS was also affected by cross-linguistic differences. We segment below six utterances of an artificial language of our sample (Experiment 2, corpus 1), to illustrate how TP and DiBS were sensitive to morpheme boundaries. Word boundaries are marked with a space and morpheme boundaries are marked with a hyphen for readability. Here are the 
six utterances with gold segmentation:

- yu tihu-gu

- su-gu

- gu rugeza-pe

- yu huyahori-ge

- tupe-gu

- ga guyufiqu-zi

FTPa segmented this as follows:

- yu tihu gu

- sugu

- gu ruge zape

- yu hu yahorige

- tupe gu

- gagu yu fiquzi

TP segmented out the affix "gu" in two cases, after measuring the transitional probabilities across each syllable pair, and comparing to the average threshold. For example, $\operatorname{TP}($ hugu $)=1 / 2$ and $\operatorname{TP}($ pegu $)=1 / 2$. The average threshold is 0.73 , so a boundary was placed in the middle of these two syllable pairs. Since morphemes are usually combined with different stems, their transitional probabilities are low and they may be segmented.

Here is the DiBS output:

- yu tihu gu

- su gu

- guru gezape 
- yuhu yahori ge

- tupe gu

- gagu yufiquzi

DiBS also segmented the affix "gu" in three cases. This may be attributed to the fact that the onset of the particular morpheme was also the first onset in some utterances. Thus, models making use of surface cues can also be affected by morphological complexity.

Finally, both baselines did a better job segmenting Japanese than Chintang on the word level. However, results were different on the morpheme level. Chintang morphemes are on average shorter than Japanese ones (according to Table 1, Chintang morphemes are often one-syllable long), whereas the opposite is true for words. As a result, on morphemes, performance is very high when boundaries are systematically posited after every syllable (Syll=Unit), and very low when no boundary is posited at all $(\mathrm{Utt}=\mathrm{Unit})$. This might explain why, when evaluated on morphemes, Chintang results outperformed Japanese ones with Syll=Unit, but were lower than Japanese ones with Utt=Unit.

Last, all algorithms yielded lower performance for our natural and artificial languages than what is previously documented for English. For example, TP seems to work better in finding word boundaries for languages with monosyllabic words, such as English, than for the languages of our sample. This might mean that implementing these segmentation strategies works well for English, but might need adaptation for other linguistic systems (see M. Johnson et al., 2007 for an adaptation of AG to Sesotho). It might also mean that English is a particularly easy language to segment (see Fourtassi et al., 2013 for such a claim), or that some algorithms are less informative in other languages (see Overall discussion for specific hypotheses). 